\title{
Être influenceur voyage et maintenir l'envie du monde en temps de pandémie
}

Being Travel Influencers and Maintaining the Desire to Travel During the

Pandemic Onset

\section{Eugénie Pereira Couttolenc}

\section{CpenEdition}

\section{Journals}

Édition électronique

URL : https://journals.openedition.org/tourisme/4002

DOI : $10.4000 /$ tourisme.4002

ISSN : 2492-7503

\section{Éditeur}

Association Mondes du tourisme

\section{Référence électronique}

Eugénie Pereira Couttolenc, «Être influenceur voyage et maintenir l'envie du monde en temps de pandémie », Mondes du Tourisme [En ligne], 20 | 2021, mis en ligne le 15 décembre 2021, consulté le 17 décembre 2021. URL : http://journals.openedition.org/tourisme/4002 ; DOI : https://doi.org/10.4000/ tourisme.4002

Ce document a été généré automatiquement le 17 décembre 2021.

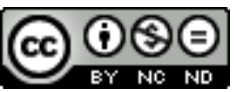

Mondes du tourisme est mis à disposition selon les termes de la licence Creative Commons Attribution - Pas d'Utilisation Commerciale - Pas de Modification 4.0 International. 


\title{
Être influenceur voyage et maintenir l'envie du monde en temps de pandémie
}

\author{
Being Travel Influencers and Maintaining the Desire to Travel During the \\ Pandemic Onset
}

Eugénie Pereira Couttolenc

\section{Introduction}

Dans cet article, nous adoptons le regard d'une analyste de discours pour documenter l'évolution des comportements sociolangagiers et les représentations individuelles ${ }^{1} \mathrm{de}$ huit animateurs de blogs voyage populaires sur le web survenue au cours des premiers mois de la pandémie de la Covid-192. Comment, dans une situation cumulant effondrement du marché touristique, restrictions des déplacements et climat anxiogène, les blogueurs maintiennent-ils dans leurs productions le désir de voyager? Comment les paradigmes ${ }^{3}$ qui caractérisent les discours tenus dans le champ des voyages sont-ils maintenus dans les stratégies de captation ${ }^{4}$ ? Quels sont les positionnements énonciatifs adoptés par les blogueurs dans les billets traitant du coronavirus? Sont-ils différents de ceux utilisés pour décrire habituellement les destinations touristiques?

2 L'angle conceptuel de l'analyse du discours consiste à mettre en relation les productions langagières d'un champ discursif $^{5}$ avec leurs conditions sociales d'apparition ${ }^{6}$. On rejoint ainsi l'hypothèse selon laquelle on pourrait expliquer les phénomènes sociaux à partir des représentations collectives véhiculées dans une société, des actions qu'elles autorisent (Moscovici, 2003) et des discours qui les portent (Charaudeau, 1997). Les pratiques langagières du champ des voyages ${ }^{7}$ ont été étudiées dans des disciplines telles que la communication (Boyer et Viallon, 1994) et la sociolinguistique (Dann, 1996). En analyse du discours, les études portent essentiellement sur des brochures, des dépliants ou des guides (Baider, Burger et 
Goutsos, 2004; Mourlhon-Dallies, 1995). Elles soulignent l'emploi de procédés linguistiques de valorisation pour décrire un lieu dans des brochures touristiques (Kerbrat-Orecchioni, 2004); elles décrivent, sous l'angle du genre discursif ${ }^{8}$, des guides très médiatisés tels que le "Guide du Routard " et le "Guide Gallimard » (Seoane, 2012) ; elles retracent les manifestations de l'intertexte " récit de voyage » et " guide touristique » dans les blogs (Deseilligny et Angé, 2011). D'autres publications, cette foisci anglophones, explorent également les liens entre tourisme et discours. L'ouvrage Discourse, Communication and Tourism (Jaworski et Pritchard, 2005) réunit des contributions qui mettent en avant des thèmes récurrents dans les productions touristiques: O'Reilly décrit le paradigme du discours antitouristique qui oppose la figure du touriste à celle du voyageur dans la construction identitaire des backpackers (O'Reilly, 2005) ; Kennedy observe l'exaltation des commentaires dans les écrits des cartes postales (Kennedy, 2005) ; Urry souligne comment le regard s'approprie une destination (Urry, 2005). Dans ses recherches, Azariah, quant à elle, étudie la manière dont les images discursives du voyageur et du touriste se côtoient, s'affrontent et s'associent dans les photographies et dans les récits de présentation de soi des auteurs de blogs (Azariah, 2017). Notre démarche s'inscrit dans la lignée de ces travaux. Elle interroge la façon dont les discours, en tant que systèmes de signification propres à une configuration sociale et historique, construisent le champ des voyages et s'inscrivent dans la situation de communication de cet espace numérique. Selon cette orientation et pour documenter les éventuelles répercussions de la pandémie, on compare, dans cet article, les résultats de l'étude descriptive et qualitative des productions de neuf blogueurs, publiées entre mars 2020 et janvier 2021 et mentionnant ou traitant explicitement de l'épidémie Covid-19, à celles d'un corpus antérieur portant sur les modalités de l'invitation au départ au sein de ces mêmes travel blogs.

3 Après avoir brossé le portrait social et discursif des auteurs qui s'imposent en tant qu'influenceurs voyage dans les réseaux sociaux, nous proposons un rappel des principales lignes de tension symboliques qui façonnent les positionnements dans ce champ. Nous décrivons ensuite la façon dont les blogueurs réinventent (ou non) leurs pratiques de publication et de financement pour affronter la situation sanitaire, avant de conclure en faisant état des procédés langagiers et visuels utilisés pour maintenir, en dépit du contexte, les effets de l'enchantement propre à la rhétorique du voyage (Réau et Poupeau, 2007).

\section{Influenceurs voyage, blogs et caractéristiques sociolangagières}

4 L'influence est une notion actuellement très populaire dans les approches gestionnaires (Vaublanc, 2019). Le concept n'est pourtant pas récent en sciences sociales. Le bouche à oreille investit de nouvelles formes dans les interactions du web, permettant à de nouveaux acteurs, situés hors des circuits traditionnels de l'industrie touristique, de formuler conseils et recommandations.

\section{Qui sont les influenceurs voyage?}

Huyghe rappelle que l'influence est un terme initialement employé au XIII siècle, en astronomie, dont l'usage désigne l'incidence des agissements d'une personne sur les 
opinions et sur les croyances d'une autre pour lui faire adopter le comportement attendu (Huyghe, 2009). L'influence est un " pouvoir sur un pouvoir» (op. cit., p. 142) qui feint le jeu du libre arbitre mais n'en possède que les traits superficiels, sa finalité étant d'orienter les conduites. Son action renforce des images préexistantes ou en propose d'autres qui, à leur tour, « suscitent des manières d'être-au-monde » (Juillet et Racouchot, 2012, p. 165). Ainsi, l'art de s'adapter aux attentes et « aux cadres mentaux de l'influencé » (Huyghe, 2009, p. 146) est un prérequis pour fédérer autour du leader d'opinion une collectivité de "je" pluriel et singulier en un "nous" homogène. L'aspiration à s'échapper d'un quotidien perçu comme aliénant par le voyage (Amirou, 1995; Graburn, 1983) est la clé qui conduit à transformer une communauté d'internautes en de potentiels suiveurs de tendances (Erner, 2020). Chaque partage d'expérience devient alors un exemple à suivre et un contenu à reproduire.

6 Les influenceurs voyage sont des intermédiaires de la communication touristique d'un nouveau genre, numérique ici. Ce sont des Youtubers (Anil B ${ }^{9}$ explore des lieux de mémoire), des Instagramers (Lilyrose ${ }^{10}$ publie des photos de ses escapades) et des blogueurs (Bruno Maltor ${ }^{11}$ anime le blog Votre Tour du Monde depuis 2012). Leur "poids » dans les réseaux se mesure en nombre d'abonnés. Les micro-influenceurs comptabilisent entre 1000 et 100000 individus suiveurs (Bayle, 2019) et se distinguent des experts en influence qui disposent de plusieurs millions de membres dans leurs communautés ${ }^{12}$. À côté de ces quelques stars du web, la majorité des globetrotteurs qui partagent leur vécu en ligne le font auprès d'un auditoire plus restreint au sein de la blogosphère. Les activités de publication débutent souvent à partir des interfaces du blog et s'étendent, dans une seconde phase, à d'autres espaces de partage (Facebook, Twitter, TikTok, Snapchat, Pinterest). L'image du voyageur, mise ainsi en réseaux, permet aux influenceurs d'interpréter plusieurs rôles, de s'adresser à des publics différents et d'adapter leurs messages aux pratiques de navigation. Tandis que le blog fournit des informations détaillées sur les destinations visitées, Instagram souligne l'aspect visuel et esthétique du voyage et YouTube soumet au public des vidéos dynamiques et ludiques des lieux à explorer. Récits, photos et vidéos établissent conjointement la véracité du témoignage de l'auteur et lui donnent autorité pour formuler des conseils (Pereira Couttolenc, 2021).

Plus spécifiquement, le blog voyage nous intéresse en ce qu'il est un lieu d'échange où un amateur (Flichy, 2010) partage des expériences personnelles de séjours de courte ou de longue durée en France et à l'étranger. Son auteur n'est ni détenteur d'un savoir attesté ni bénéficiaire de l'appui d'une instance de légitimation, comme le serait un collectif de guides adoubé par une grande maison d'édition. Pour attirer et retenir l'attention des internautes (Citton, 2014) et pour inciter ses lecteurs à entreprendre un voyage, le blogueur doit avant tout établir sa légitimité. Les sections du blog «À propos" et "Qui suis-je?" ainsi que les nombreux billets postés permettent à l'influenceur d'endosser successivement les habits linguistiques de l'éditeur, du narrateur, du témoin, de l'évaluateur, du guide, du voyagiste et du complice, dévoilant ainsi une connaissance importante des comportements sociodiscursifs de l'ensemble des acteurs du tourisme. Son statut de béotien n'est donc qu'apparent. 


\section{Les compétences sociodiscursives des influenceurs voyage}

8 L'exploitation des résultats de notre première étude a établi que, pour asseoir sa posture, le blogueur doit développer de nombreuses compétences. L'auteur doit, avant tout, mobiliser des outils d'ordre éducatif puisqu'il est nécessaire de savoir rédiger un texte numérique construit, illustré et argumenté et de produire un à trois billets par mois pour maintenir l'intérêt de ses lecteurs. Ses aptitudes relèvent également de la maîtrise technique de l'interface : animer un blog et maintenir la fidélité de son réseau requièrent l'acquisition de langages informatiques et supposent d'être initié au fonctionnement des moteurs de recherche (Cardon, 2015 ; Thiers et Ringard, 2019). De surcroit, le blogueur se doit de connaître les schèmes ${ }^{13}$ discursifs et sémiotiques habituellement employés dans le domaine touristique pour réinvestir, dans ses narrations, les traits langagiers et visuels des autres genres de discours habitant le champ des voyages. Ces éléments s'observent notamment dans la reprise des intitulés « à voir » et « à faire » propres aux guide pratiques; dans l'utilisation du temps présent dans un récit au passé, caractérisant les reportages de la presse touristique; dans la proposition de lieux d'hébergement renvoyant, plus traditionnellement, aux activités des voyagistes, etc.

9 La maitrise de l'ensemble de ces scripts se note, par ailleurs, dans les mises en scène des paradigmes constitutifs du domaine. L'aspiration à convaincre les lecteurs de l'authenticité de la démarche et de l'expérience proposée (MacCannell, 2013; Cousin, 2011) s'observe aussi bien dans les photos (Azariah, 2017) que dans les nombreux emplois du champ sémantique du Vrai (Boyer, 1996 ; MacCannell, 2013) ou encore dans la mobilisation récurrente d'une écriture vive qui recrée les effets d'une communication immédiate (Rosier, 2006). Le registre familier du blogueur contraste avec les tournures formelles des acteurs de la promotion touristique. L'humour, les phrases exclamatives et les émoticônes simulent la complicité entre auteur et lecteurs. Dans les narrations, les conseils proposés pour visiter des lieux très prisés sans se voir submerger par les vagues de touristes et les invitations formulées pour voyager hors des sentiers battus (Gravari-Barbas et Delaplace, 2015) réactivent, à la faveur de l'auteur, le scénario de la révélation (Urbain, 2002). L'image de l'auditoire est ici celle d'un lecteur qui se voit confier, dans l'intimité relative des publications du blogueur, les clés pour devenir à son tour un explorateur des temps modernes. La parole partagée concoure à forger les attentes et les itinéraires du futur voyageur (Boorstin, 1992). À ce paradigme, il faut encore ajouter celui de l'accord tacite qui lie blogueurs, lecteurs et professionnels du tourisme autour d'« un déni des caractéristiques marchandes des services » (Réau et Poupeau, 2007, p. 10) et qui les unit dans leur aspiration à entretenir «une relation enchantée au monde»(ibid.). Les influenceurs décrivent, par de multiples évaluations positives, les lieux visités et ne mentionnent que très peu les aspects négatifs rencontrés. De la même manière, les commentaires postés par les internautes ne sont, le plus souvent, qu'éloges et remerciements. Les compétences sociales et discursives des blogueurs renforcent leur légitimité à conseiller d'autres internautes. La connaissance des scripts et des routines du domaine suppose, aux yeux des futurs voyageurs, une expertise acquise sur le terrain. Cette supposition est d'autant plus ancrée chez les lecteurs que les photos publiées attestent du vécu de l'auteur (Sontag, 1977). 


\section{La dimension iconographique des blogs voyage}

10 Tourisme et photographie concourent ensemble à la narration du voyage et à l'identité discursive que donne à voir le voyageur dans ses récits (Azariah, 2017; Baerenholdt, 2004 ; van Dick, 2008). Dans la textualité particulière du blog, la photo légitime le blogueur dans son rôle de conseiller tout autant qu'elle invite au voyage (KerbratOrecchioni, 2004). Les récents développements technologiques facilitent grandement la diffusion des images sur les réseaux sociaux (van Dijck, 2008). Or, on le sait, l'appropriation des lieux touristiques (Urry, 1990) fait partie d'une herméneutique fermée où l'image capturée doit refléter celles vues dans les médias (brochures, guides, magazines, reportages télé...) avant d'être diffusée à son tour dans la sphère privée ou publique (Jenkins, 2003; Urry, 1990). Il est à noter que la mise en scène visuelle de l'objet photographié dans les blogs voyage ne s'arrête pas à la seule dimension esthétique, elle réinvestit également les scripts sémiotiques employés dans les supports promotionnels du secteur touristique (Azariah, 2017). On retrouve ainsi les marottes des voyagistes dans les articles des blogueurs, telles que la vue d'un soleil couchant ou des étendues de sable blanc (Dann, 1996) ou encore l'absence d'individus sur les photos. La pertinence de la distinction entre les actes de l'amateur (Robinson et Picard, 2009) et ceux du professionnel (MacCannell, 2013) a peu d'intérêt dans l'espace du blog voyage où les logiciels retravaillent les clichés et se mettent au service des intentions de séduction du photographe (Azariah, 2017). Dans tous les cas, les photos nous racontent quelque chose de l'expérience du voyage passé (van Dijck, 2008) et de celui à venir. Elles contribuent à l'élaboration du sens attribué au voyage et participent de la relation qui s'établit entre les influenceurs et leurs audiences.

\section{Blogs voyage et financements}

11 Du point de vue économique, l'étude des hyperliens, comme lieu d'inscription d'une énonciation double (celle du blogueur et celle d'un acteur de l'industrie touristique), a révélé que les blogueurs ont une conscience aiguë du potentiel marchand de leurs activités de création de contenu et de leurs recommandations (Pereira Couttolenc, 2020). On assiste alors à une professionnalisation de la démarche, à travers laquelle chaque intervenant peut se convertir en relais de communication des acteurs du marché. Tandis que quelques blogueurs affirment leur indépendance ${ }^{14}$, il n'en demeure pas moins que d'autres recourent à différentes options pour rémunérer leurs activités. D'aucuns développent des produits qu'ils commercialisent sur leur site : ce sont par exemple des applications de géolocalisation ${ }^{15}$, des e-books pour organiser un tour du monde ${ }^{16}$ ou des livres à acquérir ${ }^{17}$. Fréquemment, quand les blogueurs attirent dans leur toile un grand nombre d'abonnés, la tentation de tisser des partenariats avec des annonceurs institutionnels ou privés (comme les plateformes de comparaison ou de réservation en ligne, les agences régionales de promotion du tourisme...) est forte. Le sponsoring consiste soit à être mandaté par un tiers pour réaliser un reportage photo soit à associer, dans le corps du texte, des hyperliens aux noms des hôtels, des restaurants et des loisirs recommandés. La pratique semble délicate pour les influenceurs. Il leur faut maintenir l'image d'une voix authentique, hors des chemins de l'industrie touristique, tout en tirant profit des longues heures qu'ils investissent dans la création de contenu. Pour justifier ces alliances, les auteurs revendiquent des jugements neutres et découragent les partenariats dont les actions ne paraissent pas 
correspondre à leur ligne éditoriale ${ }^{18}$. La biosphère des blogs voyage est donc hétérogène en matière de rémunération et, concrètement, très peu de blogueurs parviennent à vivre des gains qu'ils tirent de leurs activités ${ }^{19}$.

Il importe à ce stade de détailler la manière dont nous avons procédé pour mettre à jour ce type de tensions dans la matière plurisémiotique des blogs.

\section{Corpus et entrées d'analyse}

13 Le premier corpus se compose d'un ensemble de huit blogs voyage dont la quête de visibilité est clairement affichée. Dans le contexte numérique, les énoncés pris en compte pour l'analyse relèvent du langagier et du plurisémiotique (Paveau, 2015). Par conséquent, nous avons rassemblé entre 2016 et 2019 une soixantaine de billets, 300 commentaires et une centaine de photos. Pour pallier le fait que les résultats de cette recherche proviennent de données collectées dans une conjoncture sociohistorique distincte de celle que nous vivons depuis 2020 , ce recueil a été complété d'un ensemble additionnel composé des publications de neuf blogs ${ }^{20}$ parues entre le 15 mars 2020 et le 15 janvier 2021 (cf. illustration 1 ).

14 Pour analyser les documents réunis, nous avons considéré que la situation de communication mise en place dans le blog conduisait l'influenceur à devoir conquérir le droit à la parole (Charaudeau, 1995). Pour convaincre son public, nous l'avons mentionné, le blogueur doit non seulement réinvestir les codes langagiers et plurisémiotiques qui caractérisent les productions du champ des voyages mais il doit également relever le défi que le genre du blog pose : incarner une parole vraie. Afin de caractériser les représentations que les auteurs donnent à voir d'eux-mêmes, de leurs interlocuteurs et du thème qu'ils dépeignent dans la textualité particulière du blog, nous avons adopté une démarche méthodologique alliant analyse du discours (Maingueneau, 1984, 2013, 2014; von Münchow, 2007, 2016) et analyse sémiotique (Kress et van Leeuwen, 2006). Les deux disciplines se font écho dans leurs approches puisque toutes deux interrogent la façon dont les narrateurs interprètent et mettent en scène leurs expériences, dans une perspective critique (op. cit.). Suivant cette direction, nous avons relevé les «traits langagiers » et « visuels » présents « dans les documents qui constituent le corpus » (von Münchow, 2007, p. 126) afin de reconstituer les images véhiculées sur les thèmes traités et les interlocuteurs visés. Nous avons, entre autres, relevé les marques de la prise en charge énonciative des locuteurs (Maingueneau, 2013); nous avons identifié les procédés linguistiques mobilisés pour qualifier les lieux recommandés (Charaudeau, 1992); nous avons été attentifs aux modalités d'appréciation déployées pour caractériser les objets de discours (Ibid. ; Moirand, 1990); nous avons souligné les formes d'adresse et les désignations référant aux énonciateurs mentionnés explicitement ou implicitement dans la matière des blogs (Maingueneau, 2013) ; nous nous sommes intéressée aux actes de langage (Kerbrat-Orecchioni, 2016) actualisés dans les productions et aux formules de justification qui apparaissent parfois dans les textes ou dans les commentaires; nous avons également explicité le type et le rôle des hyperliens dans l'élaboration des récits; enfin, nous avons décrit les clichés du corpus en nous attachant à dépeindre les relations entre participants présents et représentés selon les critères préconisés dans la grammaire visuelle de Kress et van Leeuwen (2006) (exemple d'analyse présenté à l'illustration 8b). La comparaison des résultats obtenus à partir du traitement des deux corpus a permis de mettre en 
exergue la façon dont les blogueurs ont fait face aux effets de la pandémie durant la période d'observation.

\section{Présentation des résultats}

La pandémie affecte l'ensemble du secteur touristique ${ }^{21}$. L'Organisation mondiale du tourisme soutenait en janvier 2021, que 100 à 120 millions d'emplois étaient menacés de suppression, essentiellement dans les petites et moyennes structures ${ }^{22}$. Or, peu d'études, pour l'heure, documentent les conséquences sociales et économiques de la Covid-19 sur la vie de ces individus dont l'activité (formelle ou informelle) dépend des aléas de l'industrie ${ }^{23}$.

\section{Être confiné et s'adapter} crise sanitaire. Tout d'abord, ils s'adaptent. Les blogueurs s'attachent à poursuivre leur travail de création soit par la dynamisation de contenus existants soit en détournant la signification du voyage pour l'amener hors de ses frontières traditionnelles. des blogueurs ne traitent généralement que d'un thème à la fois. Par exemple, les Bestjobers ${ }^{24}$ proposent à leur lectorat de s'inspirer de leur séjour dans une yourte au cœur du Morvan et Nuage Nomade fait le récit de sa découverte de la ville médiévale de Česky Krumlov ( $c f$. illustrations 2a et 2b). Face à l'immobilité qu'impose le confinement, les auteurs exploitent sous un autre angle des destinations qu'ils ont précédemment commentées. Un ensemble de billets distincts peut alors être réuni dans un article de synthèse ou dans une vidéo. Cette pratique, fréquente pour valoriser d'anciennes parutions, est davantage mise en œuvre dans le second corpus. Les Bestjobers revisitent ainsi leurs meilleures expériences de logement dans des cabanes (Bestjobers, 03/05/2020; cf. illustration 3a). Maman Voyage projette l'organisation de ses prochaines vacances dans un article recommandant " 10 hébergements insolites en famille» (Maman Voyage, 15/06/2020 ; cf. illustration 3b). Le Blog de Mathilde publie, quant à lui, un bilan sur la diversité des rencontres que l'on fait lorsqu'on est guide (Le Blog de Mathilde, 09/04/2020). Par ailleurs, les blogueurs sont réceptifs aux inquiétudes de leurs followers. Ils créent des articles sur les possibilités de voyager ou non en temps de pandémie (Le Sac à Dos, non daté ; Gaijinjapan, 08/06/2020; cf. illustration 4a). Ils s'attardent à décrire la mise en place des nouvelles mesures sanitaires pour voyager : prendre l'avion avec un masque, remplir des attestations, suivre les règles propres à un pays (Maman Voyage, 19/07/2020 ; cf. illustration 4b). De nouveaux sujets surgissent, comme le thème de l'assurance annulation, qui devient récurrent (Bestjobers, 02/04/2020) ou celui du remboursement des billets d'avion (Le Sac à Dos, 07/03/2020 ; cf. illustration 5).

18 Les restrictions qui pèsent sur les déplacements poussent les créateurs de contenu à explorer les frontières naturelles du voyage pour trouver de nouvelles inspirations. Le reportage ne se centre plus uniquement sur le déroulement du séjour touristique. Il s'invite, par exemple, dans les habitats sous la forme du home tour virtuel. Certains influenceurs ouvrent la porte de leur intimité et réalisent des posts sur leur décoration d'intérieur (Le Blog de Mathilde, 13/04/2020; Bestjobers, 01/05/2020; cf. 
illustrations $6 \mathrm{a}$ et $6 \mathrm{~b})$. Les objets les plus ordinaires deviennent les témoins d'un style de vie qui consiste à "endosser la condition de voyageur à chaque instant du quotidien" (Calefato, 2018, p. 148). Dans le titre "Home tour: dans la casa des Bestjobers!", les mots d'emprunt, anglais et espagnol, nous laissent imaginer des déambulations dans un espace plurilingue distinct et distant de celui de notre jour à jour de confinés. Ces procédés sémantiques revêtent d'exotisme les descriptions les plus simples. L'habillement devient un autre sujet fréquent tandis que, dans les faits relevés entre 2016 et 2019, seuls les emblèmes du baroudeur, l'équipement photo et le sac à dos, étaient présentés aux lecteurs (Bestjobers, 07/09/2016 ; Le Sac à Dos, réédité le 20/12/2020).

19 Lorsque les restrictions se lèvent, les blogueurs proposent à leur auditoire de s'évader vers des destinations ouvertes à l'arrivée des Français de métropole : la Martinique et la Corse pour les Bestjobers; Dubaï et les îles Canaries pour Un Couple en Vadrouille. Ils suggèrent de (re)découvrir les régions de France d'une façon originale, en canoë ou à vélo... L'objectif du photographe donne à des lieux, auparavant boudés, des airs de territoires inexplorés. On s'émerveille, seul à Verdun, face à la grandeur des bâtiments, et Bar-le-Duc s'offre aux prises de vue en surplomb (Un Couple en Vadrouille, $28 / 10 / 2020$ et $09 / 12 / 2020$; cf. illustration 12 ). Les auteurs créent également des itinéraires qui respectent la limite des $100 \mathrm{kms}$ ou ils suggèrent des manières de voyager autrement, sans hôtel ni restaurant (c'est l'exemple de la Vanlife des Bestjobers - Bestjobers, 24/06/2020). Le statut du traitement de l'épidémie évolue au fil des mois dans les articles, pour se convertir en une information supplémentaire à prendre en compte « avant de partir » et s'insérer plus discrètement dans des encadrés dédiés aux préparatifs ( $c f$. illustration 13).

20 Les blogueurs l'ont bien compris : les façons de se déplacer peuvent changer ou bien être limitées mais l'envie du monde (Urbain, 2018) reste présente. Les influenceurs saisissent également avec acuité la nécessité de déployer des stratégies de maintien de l'attention auprès d'une audience qu'ils savent volatile. Les articles de synthèse sous forme de liste, les adaptations $d u$ thème du voyage aux contraintes actuelles réactualisent, durant cette période, le contrat tacite qui lie les digital nomads aux internautes: indépendamment de leur situation personnelle ou du contexte mondial, les auteurs maintiennent le rythme de leurs publications, cultivant ainsi l'image du voyageur idéal qui s'adapte quelle que soit la nature de l'imprévu.

\section{Trouver de nouveaux financements}

21 Au début de la pandémie, le confinement est vécu comme une parenthèse. C'est un moment de pause, pendant lequel on réalise des tâches en attente. Alors que la crise sanitaire s'installe durablement, les inquiétudes des influenceurs s'accentuent. L'auteure du Blog de Mathilde partage ses craintes dès la mi-mars: "En plus de l'interdiction de voyager des Européens aux États-Unis - ce qui impacte directement mes 2 business (les visites + le blog qui vend des activités, hôtels à des voyageurs aux États-Unis), la ville de Boston prend des mesures drastiques. (...) Je me résous au fait de sans doute n'avoir aucun revenu dans les prochaines semaines" (Le Blog de Mathilde, 18/03/2020). Les Bestjobers, quant à eux, s'expriment à cœur ouvert sur leur chaîne YouTube : «Après euh : la perte économique ! euh : c'est vrai que ça fait deux mois que tout est à l'arrêt / comme on dépend bah vraiment du secteur du tourisme / des reportages / tout est fermé / donc bah : / et c'est pas prêt de repartir tout de suite non 
plus, il faudra attendre début juin l'annonce / pour nous énorme énorme incertitude sur l'avenir - quand est-ce qu'on va pouvoir retravailler, repartir en reportage, avoir des commandes photos? On garde le moral $»$ (Bestjobers, 2:02, 10/05/2020). Le ralentissement du secteur pousse les blogueurs à se tourner vers d'autres solutions de financement. Certains demandent à leur public de devenir leurs mécènes (Le Blog de Mathilde, 01/05/2020), d'autres multiplient les partenariats auprès d'acteurs du tourisme local, régional et national. Les Bestjobers s'associent ainsi au Comité régional du tourisme de la région Aquitaine et à Atout France (Agence de développement touristique de la France) pour réaliser une série d'articles dans la région : « Nous avons visité Bordeaux lors de notre road-trip en Nouvelle Aquitaine organisé dans le cadre de la campagne de communication et de relance \#CetEteJeVisiteLaFrance en partenariat avec Atout France » (Bestjobers, 27/07/2020).

Les influenceurs font ainsi preuve de résilience dans leurs pratiques. Ce n'est cependant parfois pas suffisant pour faire face à la vague de conséquences inattendues de la pandémie. Le silence des voix des blogs voyage se fait alors assourdissant.

\section{Le silence des blogs : faire comme si ou disparaître}

Federico Casalegno déplore que les récentes avancées technologiques nous aient poussés à une incessante nécessité de communiquer (Casalegno, 2006). L'auteur aspire à des espaces silencieux, à des « lieux vides qui échappent aux codes binaires, où le creux prend la place du plein » et où, dans l'échange avec l'Autre, une "pause ajoute à la phrase le signifié "je suis encore là" ». (op.cit., p.9). Or "le bruit du silence», conséquence de la propagation du coronavirus (Dequiré et Danvers, 2021, p. 3), s'est fait entendre avec une résonnance particulière dans le second corpus. Le silence n'a pas pris les traits de cet espace propice à la compréhension mutuelle que Casalegno appelle de ses vœux. Il s'est plutôt revêtu du voile discret qui semble envelopper l'histoire des crises sanitaires mondiales (Evanno et Vincent, 2021). De fait, il est surprenant d'observer que les blogs Japon Secret, The Daydreameuse, Le Sac à Dos et Un Couple en Vadrouille n'ont abordé le thème du coronavirus qu'à l'automne. La fréquence de leurs interventions s'est ralentie. S'agissait-il d'un manque d'envie ou de possibilité d'écrire? Était-il question de légitimité à intervenir sur un sujet sanitaire ? Parallèlement, deux autres blogs du corpus se sont tus courant 2020. Bien qu'on ne puisse pas, avec certitude, lier ces deux disparitions aux conséquences de la crise sanitaire (la vie des blogs étant souvent éphémère et les raisons de cesser une activité d'écriture étant nombreuses), on peut supposer que la Covid-19 a joué un rôle de catalyseur dans ces mises sous silence. Silence qui a semblé être, pour quelques-uns des influenceurs de nos corpus, la réponse la plus appropriée au contexte sociétal.

\section{Explorer des genres de discours connexes}

Au cours des mois de mars, avril et mai 2020, on observe que trois des neufs blogueurs du second corpus ont exploité des genres de discours qui favorisent le partage de confidences, tels que le récit de voyage, le journal personnel et les émissions télévisées du type entretien en face-à-face.

Ce déplacement se remarque initialement dans la composition syntaxique des intitulés des rubriques, qui reprennent les formes conventionnelles des titres de la littérature 
voyage (" journal de voyage d'un philosophe », « d'un migrant », «d'une expatriée »). Par exemple, le blog de GaijinJapan évoque le journal d'un confiné au Japon. Sur une modalité similaire Le Blog de Mathilde anime un « journal de bord en temps de covid-19 \#1 ». Les auteurs publient des résumés de leur expérience à la sortie du confinement («Fin d'état d'urgence au Japon, comment je l'ai vécu!», GaijinJapan, 03/06/2020) ou font état de leurs humeurs hebdomadaires sous un angle volontairement optimiste (Le Blog de Mathilde, 07/04/2020). Dans ces initiatives, on relève la présence d'indices langagiers empruntés au genre du récit de voyage. On constate, entre autres, l'emploi plus régulier de la première personne du singulier et du pluriel: «Déjà fin janvier, j'avais senti que cette "épidémie" [...] allait avoir un impact sur nos vies et qu'il fallait prendre quelques précautions» (Gaijinjapan, 03/06/2020). L'alternance des temps du passé ainsi que l'usage de marqueurs temporels tels que les dates, les événements et les étapes de la pandémie rythment la narration: "Dimanche 15 mars. C'est la fin des vacances. [...] On est parti il y a une semaine pour 8 jours de voile dans les Bahamas. [...] Ça ne nous semblait pas irresponsable de partir » (Le Blog de Mathilde, 18/03/2020). La dimension des émotions ressenties apparaît de façon plus saillante dans les productions: "Le coronavirus était devenu le quatrième membre de notre famille, invisible mais présent » (GaijinJapan, 03/06/2020) ; «Je crois qu'il m'a fallu un certain temps pour prendre l'ampleur de la situation. Ce n'était pas du déni, non. Juste un lent moment pour comprendre l'impact de cette situation inédite. Comme quand quelque chose de tragique arrive, on ne réalise pas tout tout de suite. Ça infuse » (Le Blog de Mathilde, 28/03/2020). Des comparaisons entre pays d'origine et pays hôte sont évoquées, mettant en contraste les mesures prises pour faire face à la pandémie : «Au Japon, il n'est pas possible d'instaurer un confinement forcé de la population, c'est illégal, au contraire de ce qui se fait à l'heure actuelle en France» (Gaijinjapan, 08/04/2020). Par ailleurs, les écrits se complètent de descriptions d'un quotidien où chacun meuble à sa façon les longues heures du stay at home. Le carnet de voyage qui fait état du confinement dépeint aussi ce temps long du trajet qui mène à destination et qu'il faut savoir occuper soit en publiant des photos en train de cuisiner, de se faire une pédicure, de faire du sport (Le Blog de Mathilde, 02/04/2020) ou de participer à des visioconférences entre amis (Le Blog de Mathilde, 28/03/2020), soit en évoquant les changements à adopter pour se rendre chez le pédiatre ou chez le coiffeur (GaijinJapan, 09/04/2020).

26 La séquence du Blog de Mathilde "What's make me happy this week ${ }^{26}$ dure pendant 25 semaines. Cette série de billets se caractérise par l'occurrence encore plus fréquente de la marque de la première personne et du champ sémantique des émotions. Il semble que, dans ce cas, l'auteure emprunte davantage aux codes du journal personnel que ses pairs. Chaque article se compose d'une liste qui fait état des différents aspects de la vie de la blogueuse durant la crise : "Alors ce qui me rend heureuse cette semaine, ce sont 6 choses en particulier - jeu en ligne, podcast, app, livre, série et musique, que je partage avec vous dans cet article » (Le Blog de Mathilde, 07/04/2020). Ces publications sont aussi un moment d'échange plus intime avec les lecteurs sur les conséquences inattendues de la crise : « certains projets de vie sont en pause également. C'est dur. On attend" (Ibid.). Les Bestjobers lancent également une rubrique plus introspective sur leur chaîne YouTube. Les auteurs s'inspirent d'une émission télévisée, "Le Divan ", dans laquelle une célébrité s'exprime sur ses choix de carrière, commente des passages difficiles de sa vie, etc. Reprenant les conventions de cette production, le couple se met en scène sur un sofa et profite de ce moment de pause pour répondre aux questions 
récurrentes des internautes (Bestjobers, 12/04/2020). Enfin, Maman Voyage expose son expérience de la pandémie en tant que mère dans un compte rendu hebdomadaire.

En exploitant des genres discursifs proches de ceux convoqués dans les univers du blog et du voyage, les blogueurs renforcent le lien qui les unit à leur communauté de lecteurs. Ce moment humanise l'image de l'influenceur qui n'est plus seulement perçu comme vivant dans le temps et dans l'espace de l'imaginaire du voyage, mais comme un individu également affecté par les conséquences de la pandémie. En se rendant plus accessible, l'auteur permet à son auditoire de se glisser derrière le rideau de la mise en scène du blog, tout en renforçant la perception d'authenticité de sa démarche. Dans cette situation particulière, ce n'est pas tant l'aspect cognitif de l'interaction qui est privilégié que sa dimension communicationnelle.

\section{Le merveilleux malgré tout}

À l'aune des résultats observés dans les deux corpus, il semble que les spécificités langagières propres à la mise en récit d'un voyage dans l'espace du blog se maintiennent en dépit des effets de la pandémie sur les situations individuelles des influenceurs et indépendamment de la variabilité de leurs réactions.

Lors de la comparaison des opérations discursives, nous avons en effet relevé des similitudes quant aux procédés de valorisation de l'objet décrit, qu'il s'agisse d'un voyage réalisé, d'un livre ou encore de l'école à distance. Par exemple, on retrouve de nombreuses évaluations appréciatives que ce soit pour recommander, avant la Covid-19, une visite à Portsmouth («c'est le plus beau bateau et le plus intéressant ! Nous l'avons visité plusieurs fois et nous ne nous en lassons pas ", Maman Voyage, 09/02/2020) ou qu'il s'agisse de décrire l'organisation de l'école à distance durant le confinement ( $«$ je suis épatée par les efforts et la qualité de ce qui est mis en place par l'école de Ticoeur et Titpuce ", Maman Voyage, 26/04/2020). On remarque également que les modalités appréciatives portant sur les lieux touristiques s'effectuent à l'identique dans les deux corpus. L'emploi de nombreux adjectifs épithètes antéposés tels que "belle» ou "beau» et l'utilisation du pluriel décrivent les atouts d'une destination (Kerbrat-Orecchioni, 2004): «Nos plus belles cabanes insolites à travers notre beau pays. [...] on vous a préparé une belle sélection » (Bestjobers, 03/05/2020). En outre, l'emploi d'euphémismes, de litotes, de tournures concessives ou encore l'ajout systématique d'un commentaire positif à toute critique négative sont des procédés qui perpétuent un effet d'euphorie permanent, que ce soit avant ou pendant la pandémie. Parfois, la répétition d'un syntagme positif dans une situation qui ne l'est pas vise tout autant à rassurer qu'à se convaincre : «Au niveau strictement personnel, ça va. Je suis en bonne santé, il y a à manger chez moi, de l'eau, de l'électricité. [...] Là, tout de suite, maintenant, ça va. Après professionnellement, c'est la catastrophe. Je perds tous mes revenus pour les prochains mois [...] Je me répète mais voilà: là tout de suite maintenant, ça $v a$, et pour le reste, c'est le gros point d'interrogation qui se mute parfois en !!!... » (Le Blog de Mathilde, 28/03/2020). Le ton enjoué propre à la rhétorique $\mathrm{du}$ voyage doit être maintenu malgré tout. Les éléments dysphoriques sont tenus à l'écart. Les blogueurs mentionnent peu d'éléments négatifs sur la situation qu'ils vivent, hormis dans quelques passages où ils expriment des sentiments de regret (« on devait»), d'incertitude (" on sait pas») et de lassitude ("encore une semaine», 
«toujours confinés »)... Parfois, aussi, c'est l'humour qui s'invite pour dédramatiser les conséquences de la pandémie, via le port du masque par exemple (cf. illustration 7).

Il faut encore noter que le pouvoir magique qui caractérise les éléments du langage touristique (Dann, 1996) perpétue ses effets sur les pratiques durant le confinement. Voici un paragraphe décrivant un livre produit par Japon Secret à l'automne 2020 :

L'éventail des lieux visités est vaste et vous serez emmenés d'un village à l'autre, qu'il soit légendaire, insolite, ordinaire, voire même abandonné. Mais ils ont tous en commun le fait d'être uniques, et les détails fascinants qui font leur charme vous donneront envie de vous y perdre. (Japon Secret, 18/11/2020)

Dans cet extrait, le champ sémantique du mystérieux ("insolite ", «légendaire ", "abandonné ») est mobilisé. Le narrateur interpelle son lecteur à la seconde personne du pluriel («vous») et l'invite à devenir unique par un acte d'acquisition. La magie de l'incantation publicitaire est à l'œuvre. En devenant consommateur, le lecteur permet au sort lancé de s'accomplir: l'internaute pourra devenir quelqu'un d'autre (Dann, 1996), un voyageur peut-être.

À partir de l'analyse sémiotique des éléments visuels, on observe également que les angles de prise de vue, le cadrage, les participants représentés (Kress et van Leeuwen, 2006), la direction des regards et les vecteurs d'action dans les photos sont identiques dans les deux corpus. Bien que la validité de nos propos repose sur les seules constatations de notre analyse contrastive, il ne semble pas qu'il y ait eu de modification majeure dans les habitudes photographiques. Ainsi, avant ou pendant la crise sanitaire, on relève que le blogueur est fréquemment représenté seul (parfois en couple) faisant face à un paysage lointain, réactivant dans les esprits, une fois encore, la figure de l'explorateur (cf. illustrations 8a, 11 et 12). Les photos de l'article du Blog de Mathilde sur l'Oregon (Le Blog de Mathilde, 15/09/2016), extraites du premier corpus, témoignent de cette représentation prégnante chez les voyageurs contemporains. Les clichés montrent une mise en scène dénuée d'encadrement logistique. L'auteure et son compagnon voyagent seuls en voiture, ils dorment visiblement à flanc de montagnes, dans des lieux à l'apparence hostile et ils organisent leur itinéraire à l'aide de cartes (cf. illustration 8a). Les photos des paysages sont souvent dépourvues, ou quasi dépourvues, de touristes ( $c f$. illustration 9). À l'instar des pratiques utilisées par le secteur marchand (cf. illustration 10), on retrouve, avant ou durant les premiers mois de la pandémie, des photos du blogueur de dos, en surplomb ou en contrebas du lieu à découvrir, dans une posture de conquérant ou de pèlerin contemplant l'incommensurabilité de la nature ( $c f$. illustrations 11 et 12). Invitant à le rejoindre par le texte et à le suivre par la route ou la rivière qu'il contemple, le blogueur s'appuie sur la mémoire des routines discursives et des images véhiculées dans le milieu viatique pour séduire l'œil du lecteur et exciter son imaginaire. Le désir de voyager s'étend audelà des limites de son chez soi, où chacun se trouve confiné, pour se prolonger, par circumnavigation, dans les méandres des photos des blogs voyage.

\section{Conclusion}

Les résultats de la comparaison effectuée sur la base des deux corpus constitués avant et pendant la crise de la Covid-19 soulignent à la fois la modification du rythme des publications, le renouvellement des thèmes abordés, la recherche de nouvelles sources de financement et certaines disparitions de blogs. La situation des influenceurs voyage 
est fragilisée. Certains choisissent de repousser, dans leurs récits, les frontières naturelles du voyage ; d'autres d'encourager un tourisme de proximité, respectueux des restrictions de déplacement. Ce n'est plus la destination qui construit la perception du voyage mais sa modalité de réalisation. Le van, le canoë, le glamping sont les traits modernes de la figure de l'explorateur. En proposant de nouveaux circuits de découverte de la France ou en développant des thématiques différentes dans leurs articles, les blogueurs perpétuent les mécanismes de distinction (Boyer, 1999) qui fondent et dynamisent les pratiques touristiques. Parallèlement, du point de vue des marques langagières mobilisées et des rôles discursifs incarnés par les auteurs des blogs, on observe que les habitudes, qu'elles soient discursives ou visuelles, se maintiennent en dépit du caractère exceptionnel de la situation sanitaire. S'il semble probable que les déplacements et les destinations des voyageurs soient affectés par les conditions sanitaires propres à chaque pays, à l'inverse, le faisceau d'indices documentés dans ce travail nous semble conforter l'avis de Jean-Didier Urbain selon lequel « les paradigmes de représentations modelées par des siècles de culture ne se modifieront pas» (Urbain, 2020, p. 63). Le désir d'ailleurs repose sur de "puissants imaginaires qui ne devraient pas s'écrouler du fait de la crise du Covid-19» (ibid.). Les pratiques photographiques perpétuant cet imaginaire en sont le témoin. Que ce soit depuis le confort de leur canapé, installés devant leurs écrans, en reportage photos ou affrontant les aléas de la pandémie, les influenceurs poursuivent leur mission, la redéfinissant parfois ou l'adaptant aux contraintes actuelles mais toujours se faisant l'écho des rêves de voyage de leurs lecteurs.

\section{BIBLIOGRAPHIE}

Rachid AMIROU, Imaginaire touristique et sociabilités du voyage, Presses Universitaires de France, 1995.

Deepti Ruth AZARIAH, Tourism, Travel and Blogging. A Discursive Analysis of Online Travel Narrative, Routledge, 2017.

Fabienne BAIDER, Marcel BURGER et Dionysis GOUTSOS (dir.), La communication touristique. Approches discursives de l'identité et de l'altérité, L'Harmattan, 2004.

Nadine BAYLE, «Les influenceurs changent la face du marketing », Le Monde, 6 octobre 2019 [https://www.lemonde.fr/economie/article/2019/10/06/les-influenceurs-changent-la-face-dumarketing_6014435_3234.html].

Jørgen Ole BAERENHOLDT, Michael HALDRUP, Jonas LARSEn et John URRY, Performing Tourist Places, Routledge, 2004.

Daniel J. BOoRSTIN, The Image: A Guide to Pseudo-Events in America, Alfred A. Knopf, 1992.

Pierre BOURDIEU, «Le champ scientifique », Actes de la recherche en sciences sociales, $\mathrm{n}^{\circ}$ 2, p. 88-104, 1976 [https://www.persee.fr/doc/arss_0335-5322_1976_num_2_2_3454].

Marc BOYER, Le tourisme de l'an 2000, Presses universitaires de Lyon, 1999. 
Marc BOYER, L'invention du tourisme, coll. « Art de vivre », Gallimard, 1996.

Marc BOYER et Philippe VIALLON, La communication touristique, PUF, 1994.

Patrizia CALEFATO, « Revêtir le voyage », Sociétés, n 139, p. 147-153, 2018.

Federico CASALEGNO, « Du silence en réseau », Sociétés, n 91, p. 49-53, 2006.

Dominique CARDON, À quoi rêvent les algorithmes. Nos vies à l'heure des big data, Seuil, 2015.

Yves citTon, L'économie de l'attention. Nouvel horizon du capitalisme?, La Découverte, 2014.

Patrick CHARAUDEAU, « Le discours de manipulation entre persuasion et influence sociale », site de Patrick Charaudeau, 2009 [http://www.patrick-charaudeau.com/Le-discours-de-manipulationentre.html].

Patrick CHARAUDEAU, Le discours d'information médiatique. La construction du miroir social, Nathan, 1997.

Patrick CHARAUDEAU, «Ce que communiquer veut dire », Revue des sciences humaines, $\mathrm{n}^{\circ}$ 51, 1995 [http://www.patrick-charaudeau.com/Ce-que-communiquer-veut-dire.html].

Patrick CHARAUDEAU, Grammaire du sens et de l'expression, Hachette, 1992.

Saskia cousin, « Authenticité et tourisme », Les Cahiers du Musée des Confluences, n 8, p. 59-66, 2011 [https://www.persee.fr/doc/mhnly_1966-6845_2011_num_8_1_1558].

Graham DANN, The Language of Tourism: A Sociolinguistic Perspective, Cab international, 1996.

Anne-Françoise DEQUIRÉ et Francis DANVERS, « Présentation », Spirale, nº 67, p. 3-7, 2021.

Oriane DESEILLIGNY et Caroline ANGÉ, « Le maillage intertextuel des blogs de voyage ou la production de figures du voyageur », Médiation et information, n³3, p. 131-140, 2011.

Guillaume ERNER, Sociologie des tendances, PUF, 2020.

Yves-Marie EVANNo et Johan VINCENT, « Tourisme et crises sanitaires mondiales dans l'Histoire : véritablement un impensé ? », journée d'études " Tourisme et pandémie : approches croisée », séminaire « Tourisme : recherches, institutions, pratiques », 4 février 2021.

Patrice FLICHY, Le sacre de l'amateur. Sociologie des passions ordinaires à l'ère numérique, Seuil, 2010.

Nelson GRABURN, « The Anthropology of Tourism », Annals of Tourism Research, n 10, p. 9-33, 1983.

Maria GRAVARI-BARBAS et Marie DELAPLACE, « Le tourisme urbain "hors des sentiers battus". Coulisses, interstices et nouveaux territoires touristiques urbains », Téoros, n 34, 2015 [https:// journals.openedition.org/teoros/2790].

François-Bernard HUYGHE, «Influence », Médium, n 18, p. 138-149, 2009.

Olivia H. JENKINS, « Photography and Travel Brochures: The Circle of Representation », Tourism Geographies, n5, p. 305-328, 2003.

Adam JAWORSKI et Annette PRITCHARD, Discourse, Communication and Tourism, Channel View Publications, 2005.

Alain JUILLET et Bruno RACOUCHOT, «L'influence, le noble art de l'intelligence économique », Communication et organisation, $n^{\circ} 42$, p. 161-174, 2012.

Chris KENNEDY, « 'Just Perfect!' The Pragmatics of Evaluation in Holiday Postcards », dans Adam JAWORSKI et Anette PRITCHARD (dir.), Discourse, Communication and Tourism, Channel View Publications, p. 223-246, 2005. 
Catherine KERBRAT-ORECCHIONI, Les actes de langage dans le discours, Armand Colin, 2016.

Catherine KERBRAT-ORECCHIONI, « Suivez le guide ! Les modalités de l'invitation au voyage dans les guides touristiques : l'exemple de l' "Île d'Aphrodite" ", dans Fabienne BAIDER, Marcel BURGER et Dionysis GouTsos (dir.), La communication touristique. Approches discursives de l'identité et de l'altérité, L'Harmattan, p. 133-150, 2004.

Catherine KERBRAT-ORECCHIONI, L'énonciation : de la subjectivité dans le langage, Armand Colin, 1980.

Thomas S. KHUN, La structure des révolutions scientifiques, Flammarion, 1972.

Gunther KRESS et Theo VAN LEEUWEN, Reading Images. The Grammar of Visual Design, Routledge, 2006.

Dean MacCANnell, The Tourist: A New Theory of the Leisure Class, University of California Press, 2013.

Dominique MAINGUENEAU, « Subvertir la distinction même entre texte et contexte », Mots. Les

langages du politique, $\mathrm{n}^{\circ} 120$, p. 185-198, 2019.

Dominique MAINGUENEAU, Discours et analyse du discours, Armand Colin, 2014.

Dominique maingueneaU, Analyser les textes de communication, Armand Colin, 2013.

Dominique maIngueneau, Genèses du discours, Pierre Mardaga Éditeur, 1984.

Sophie MOIRAND, Une grammaire des textes et des dialogues, Hachette, 1990.

Serge MoscovicI, « Des représentations collectives aux représentations sociales : éléments pour une histoire », dans Denise JODELET (dir.), Les représentations sociales, PUF, 2003, p. 79-103.

Florence MOURLHON-DALLIES, Une méthodologie pour l'analyse linguistique de genres discursifs produits en situation professionnelle : étude d'écrits touristiques sur Venise en quatre langues, thèse de doctorat, Université de la Sorbonne Nouvelle-Paris III, 1995.

Patricia von MÜNCHOW, «Quand le non-dit n'est pas l'implicite : comment rendre visibles les silences dans le discours ? », Signes, discours et sociétés, 2016 [http://revue-signes.gsu.edu.tr/ article/-LY-6XwEW9n8ye3pKT1y].

Patricia von MÜNCHOW, « Les représentations du père dans les guides parentaux français et allemands », dans Catherine SELLENET, Les pères en débat, Érès, 2007, p. 123-136.

Camille C. O'REILLY, « Tourist or Traveller? Narrating Backpacker Identity », dans Adam JAWORSKI et Annette PRITCHARD (dir.), Discourse, Communication and Tourism, Channel View Publications, 2005, p. 150-169.

Marie-Anne PAVEAU, « Ce qui s'écrit dans les univers numériques », Itinéraires, 2015 [https:// doi.org/10.4000/itineraires.2313].

Eugénie PEREIRA COUTTOLENC, « Travel Influencers », Global Encyclopedia of Informality, UCL Press, 15 mai 2021 [https://www.in-formality.com/wiki/index.php?title=Travel_influencers_(Global)].

Eugénie PEREIRA COUTTOLENC, «Liens hypertextuels et analyse des modalités énonciatives dans les discours touristiques des blogs voyage ", $23^{\mathrm{e}}$ rencontre des jeunes chercheurs, 11 juin 2020 [https://rjc2020.univ-paris3.fr/node/39].

Bertrand RÉAU et Franck POUPEAU, «L'enchantement du monde touristique », Actes de la recherche en sciences sociales, $\mathrm{n}^{\circ} 170$, p. 4-13, 2007.

Mike ROBINSON et David PICARD, The Framed World: Tourism, Tourists, and Photography, Farnham, 2009. 
Laurence ROSIER, « De la vive voix à l'écriture vive. L'interjection et les nouveaux modes d'organisation textuels », Langages, $n^{\circ}$ 161, p. 112-126, 2006.

Jean-Pierre Rossi, Psychologie de la mémoire, De Boeck Supérieur, 2005.

Annabelle SEOANE, Genre de discours et positionnements énonciatifs dans les guides touristiques : le " Guide du Routard » et le « Guide Gallimard », thèse doctorale, Université Paris Est-Créteil, 2012.

Suzan SONTAG, On Photography, Farrar, Strauss, and Giroux, 1977.

Benjamin THIERS et Julien RINGARD, Le référencement, Studyrama, 2019.

Jean-Didier URBAIN, « La crise ne va pas tuer l'imaginaire touristique ! », Espaces, n 355, 2020.

Jean-Didier URBAIN, L'envie du monde, Éditions Bréal, 2018.

Jean-Didier URBAIN, L'idiot du voyage. Histoire de touristes, Édition Payot \& Rivages, 2002.

John URRY, «The "Consuming" of Place », dans Adam JAWORSKI et Annette PRITCHARD (dir.), Discourse, Communication and Tourism, Channel View Publications, 2005, p. 19-27.

John URRY, The Tourist Gaze, Sage Publications, 1990.

José VAN DIJCK, « Digital Photography: Communication, Identity, Memory », Visual Communication, vol. 7, p. 57-76, 2008.

Géraud de VAUBLANC, Image, réputation, influence : comment construire une stratégie pour vos marques, Dunod, 2019.

\section{ANNEXES}


Illustration 1. Les blogs voyage des corpus 1 et 2 au 04/02/2021

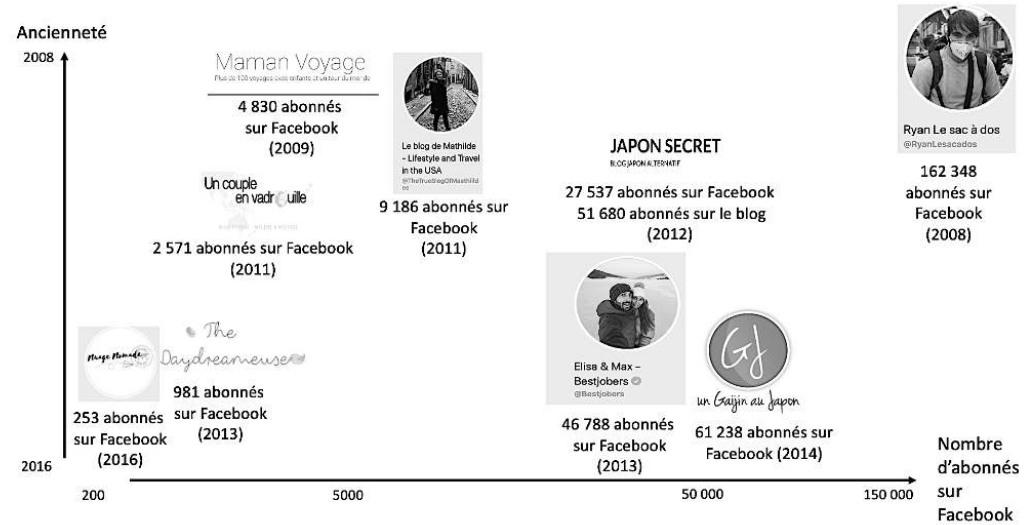

On retient les critères du nombre d'abonnés sur le réseau Facebook et celui de l'ancienneté pour caractériser la visibilité des blogs du corpus.

Liste des blogs voyage :

https://www.gaijinjapan.org/

https://japonsecret.fr

https://www.maathiildee.com/

https://lesacados.com/

https://www.bestjobersblog.com/

https://www.mamanvoyage.com/

Nuage Nomade (blog éteint en 2020)

https://www.thedaydreameuse.com/

https://www.uncoupleenvadrouille.fr

Illustration 2a. Bestjobers, « Morvan - 1 week-end presque comme au Canada à moins de $3 \mathrm{~h}$ de Paris \& de Lyon », 21 juillet 2018

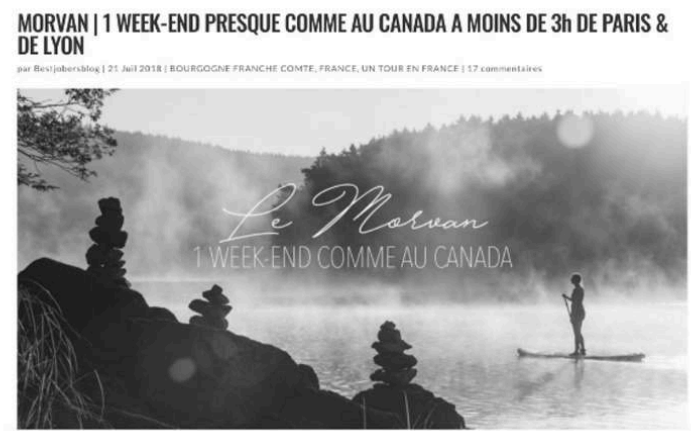

Avant dernière étape de notre \#unTOURenFrance, nous avons mis le cap sur le Morvan. Grand changement de décor après les paysages du Sud Ouest dans le Tarn et Garonne, nous voilà au coeur de la montagne bourguignonne, à mi chemin entre Paris et Lyon! Le Morvan est un vaste massif granitique, un bel écrin de verdure aux reliefs doux, fait de monts et vallées entourant de grands lacs, sculptant le paysage. La forêt est partout ou presque, feuillus et résineux couvrent la moitié du territoire. D'ailleurs nous avons appris que c'est la terre du sapin de noël avec plus d'un million d'arbres produit par an pour la France !! Si nous avions des doutes quant à sa ressemblance annoncée avec le Canada avant notre arrivée, nous avons bien dû admettre avoir été bluffés. Les immenses lacs, les forêts tout autour et toujours ce côté sauvage nous ont effectivement rappelé nos aventures en terres canadiennes! On s'est même surpris à chercher les bancs de saumon en canoë et à attendre lorignal au bord de l'eau ! Certes toute proportion gardée, c'est une chouette alternative si l'on ne peut pas aller outre Atlantique.

Source : https://www.bestjobersblog.com/morvan-1-week-end-presque-comme-au-canada-a-moinsde-3h-de-paris-de-lyon/ 
Illustration 2b. Nuage Nomade, « République Tchèque : Česky Krumlov, au cœur de la Bohème-duSud », 9 août 2017

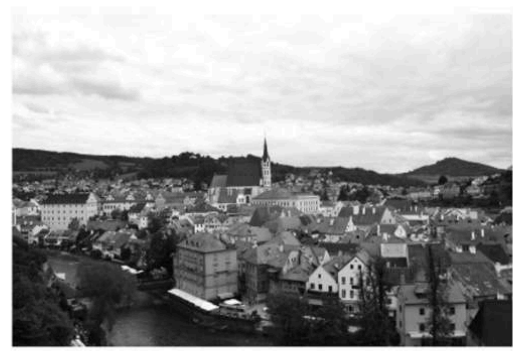

REPUBLLQUE TCHEQUE : CESKN KRUMLOV, AU COEUR DE LA BOHEME.
DU.SUD

Aujourd'hui, je vous emmène découvrir une petite ville classée au patrimoine mondial de l'UNESCO située en République Tchèque, dans la très jolie région de la Bohème-du-Sud.

Pour vous situer un peu, Český Krumlov c'est une petite ville médiévale de 13141 habitants située en Bohème-du-Sud à 85 km au nord de Linz et $123 \mathrm{~km}$ au sud de Prague. Elle est principalement connue pour son centre-ville historique qui est classé au patrimoine mondial de IUNESCO.La ville est également connue pour avoir accueilli Egon Schiele et sa compagne en 1911, il y résidera quelque temps avant de quitter la ville pour Vienne face à lhostilité de ses habitants à cause de ses peintures et son style de vie.

La source web est non disponible. Le blog ayant cessé son activité en 2020, je présente ici un extrait du travail de documentation provenant du premier corpus.

Illustration 3a. Bestjobers, « 6 cabanes insolites en France », YouTube, 3 mai 2020

$\equiv$ YouTube

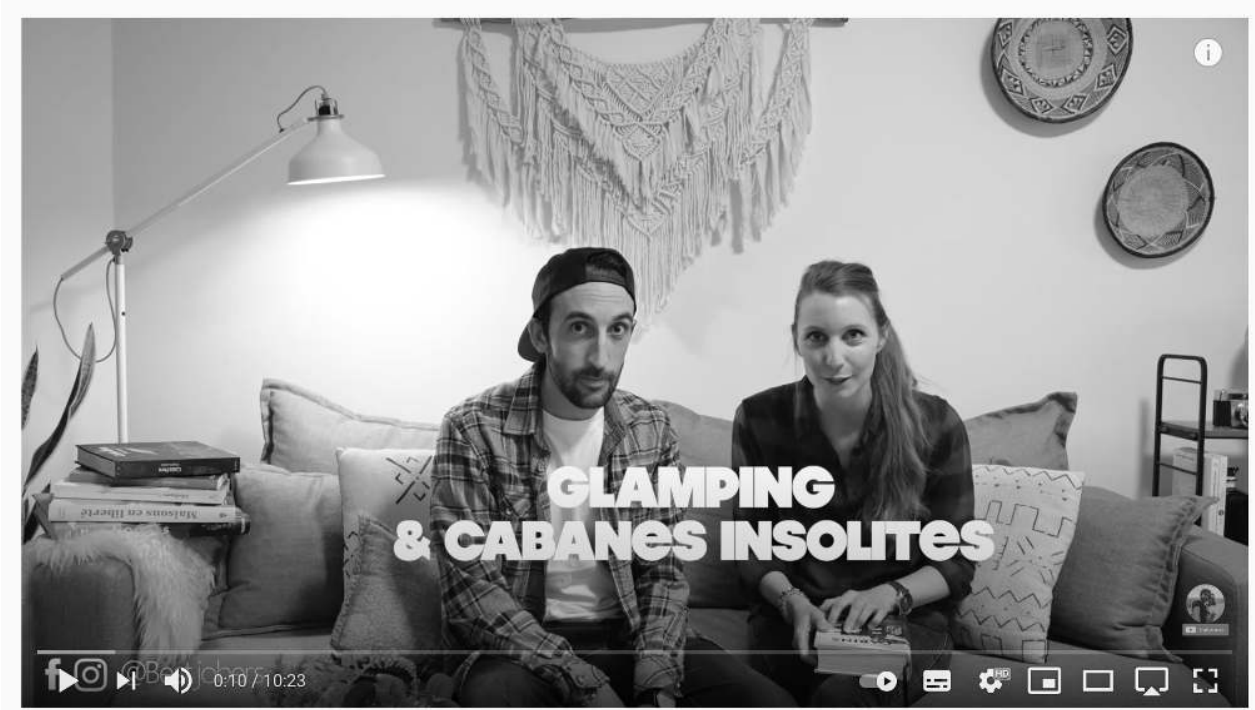

6 CABANES INSOLITES EN FRANCE I Le DIVAN des BESTJOBERS

8988 vues $\cdot 3$ mai 2020

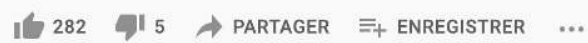

Source : https://www.youtube.com/watch?v=vAOiOmfD9ok\&t=4s 
Illustration 3b. Maman Voyage, « 10 idées d'hébergements insolites en famille », 15 juin 2020

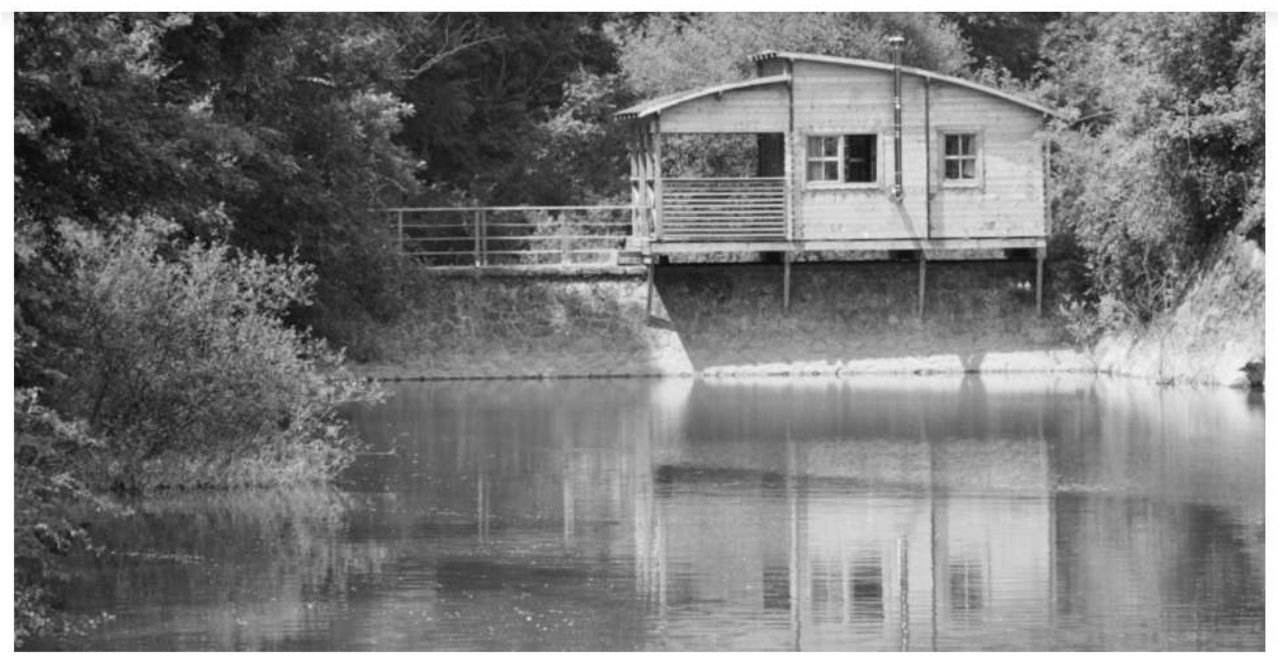

10 idées d’hébergements insolites en famille

15 juin 2020 / Nos belles adresses

Aucun commentaire

Source : https://www.mamanvoyage.com/2020/06/10-idees-dhebergements-insolites-en-famille/

Illustration 4a. GaijinJapan, « Voyage au Japon en 2020 : possible ou pas ? », 8 juin 2020

\section{VOYAGE AU JAPON EN 2020 : POSSIBLE OU PAS?}

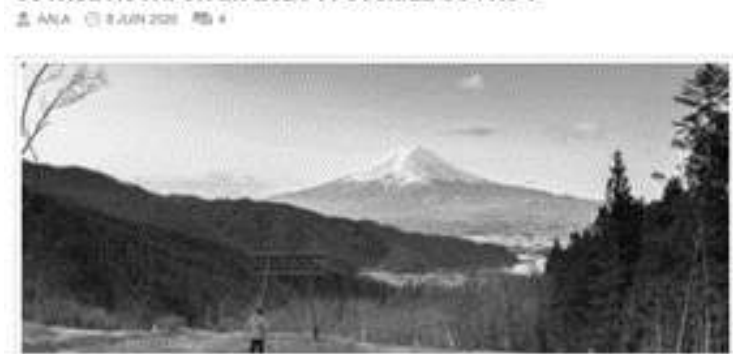

Source : https://www.gaijinjapan.org/voyage-au-japon-2020-possible-ou-pas/ 
Illustration 4b. Maman Voyage, « Prendre l'avion au temps du coronavirus », 19 juillet 2020

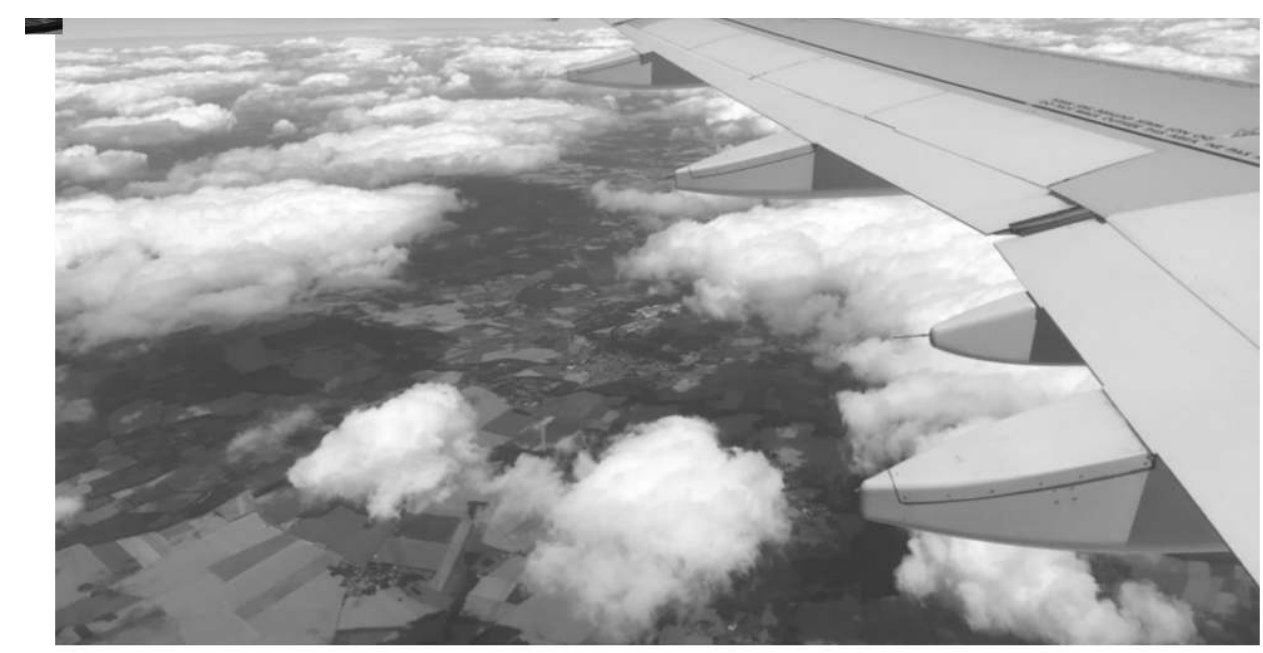

Prendre l'avion au temps du coronavirus

19 juillet 2020 / En mode voyage

10 commentaires

Source : https://www.mamanvoyage.com/2020/07/prendre-lavion-au-temps-du-coronavirus/

Illustration 5. Le Sac à Dos, "Comment demander une indemnisation pour vol retardé ou annulé ? ", 7 mars 2020

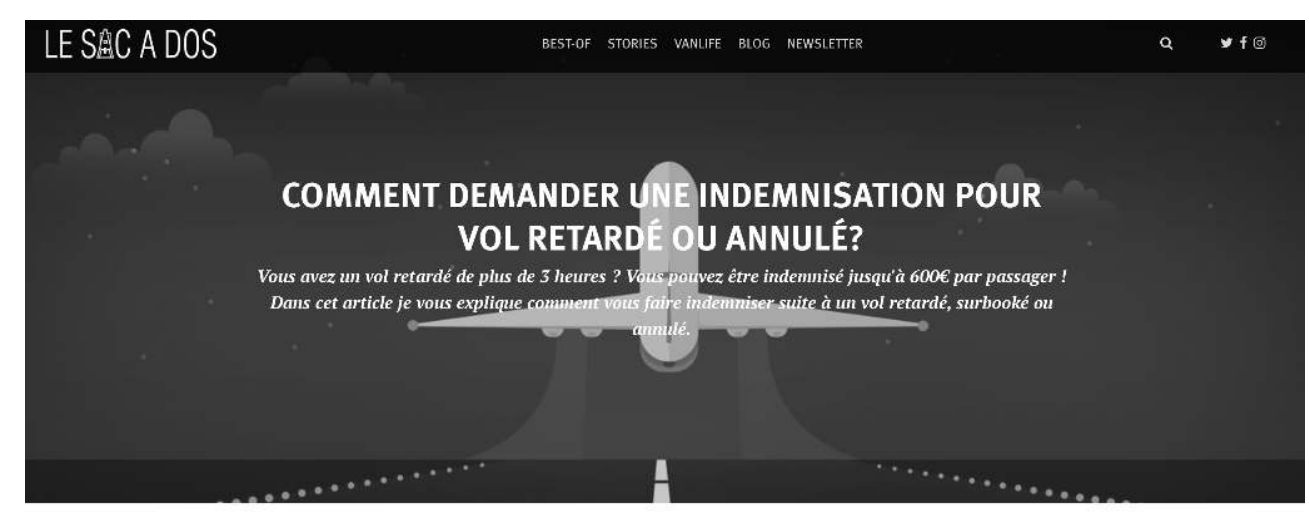

Il y a quelques temps, j’ai découvert que j'étais passé à côté de plusieurs centaines (voire milliers !) d'euros. I'ai en effet appris que la législation européenne prévoyait des droits spéciaux pour les passagers dont le vol

Source : https://lesacados.com/indemnisation-vol-retarde 
Illustration 6a. Le Blog de Mathilde, « 3 objets qui égayent mon chez moi (et leur histoire) », 13 avril 2020

E Patreon Edité le 13 Avr $2020 \oplus$ Pas de Commentaire

\section{OBJETS QUI ÉGAYENT MON CHEZ MOI (ET LEUR HISTOIRE)}

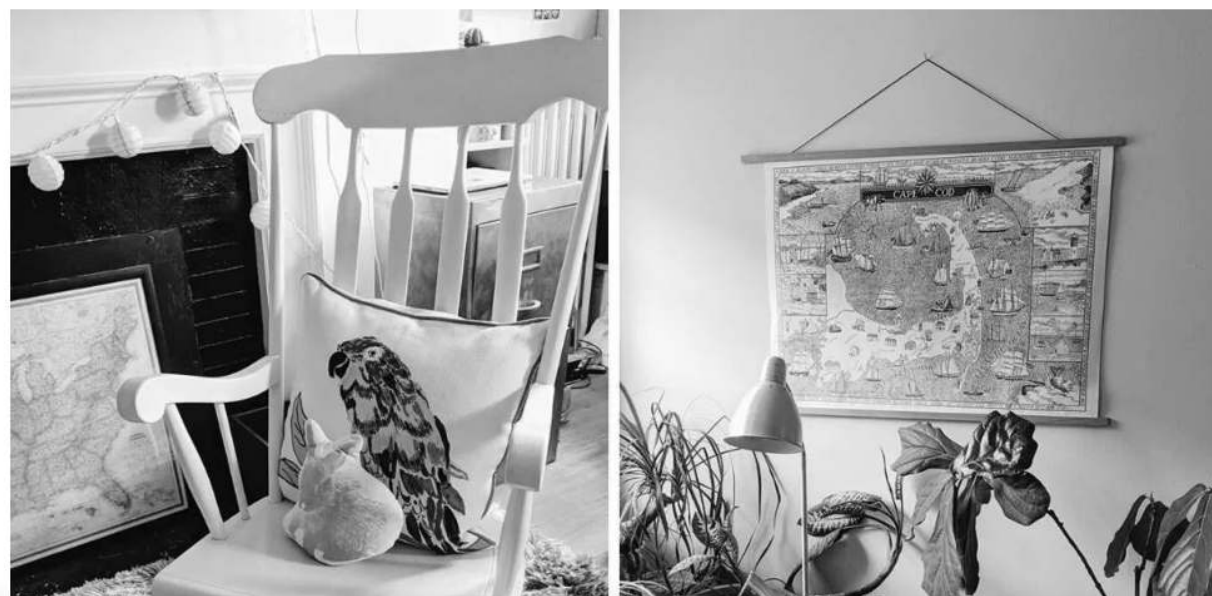

Source : https://www.maathiildee.com/3-objets-qui-egayent-mon-chez-moi-et-leur-histoire/

Illustration 6b. Bestjobers, « Home tour dans la casa des Bestjobers », $1^{\text {er }}$ mai 2020

\section{HOME TOUR | DANS LA CASA BESTJOBERS !}

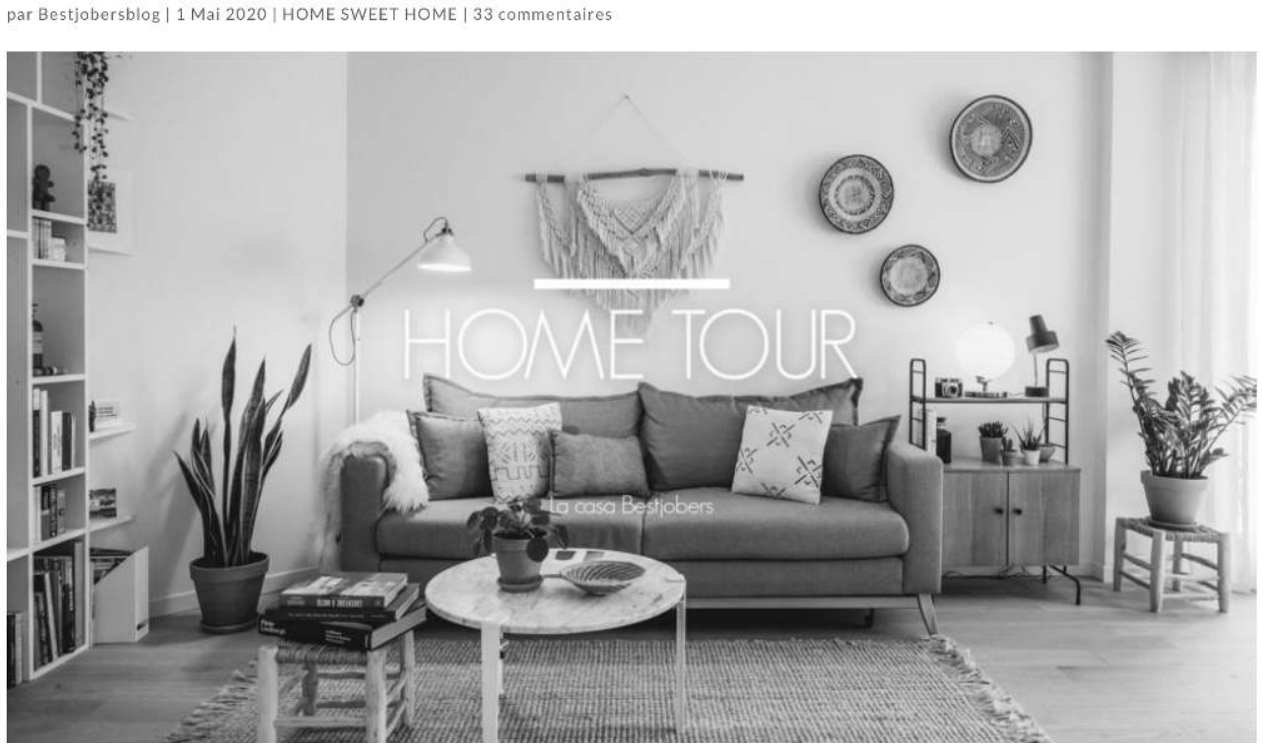

Source : https://www.bestjobersblog.com/home-tour-casa-bestjobers 
Illustration 7. Le Blog de Mathilde, "Descendre les poubelles, une nouvelle activité de couples ",

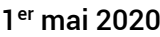

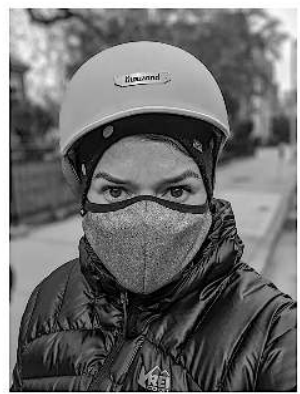

Le casque de vélo est optionnel

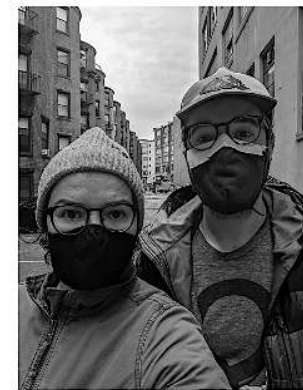

Descendre les poubelles, une nouvelle activité de couple

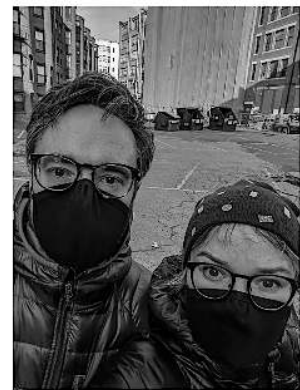

Combien de temps pour que la buée

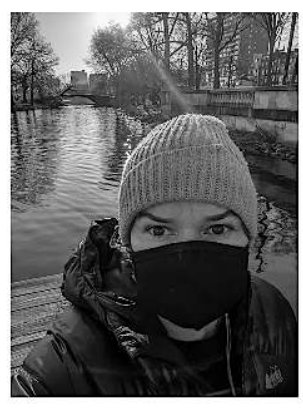

Au grand air de bon matin

Source : https://www.maathiildee.com/avril-2020-sur-le-blog-de-mathilde/

Illustration 8a. Exemples d'images extraites d'un des billets du corpus, Le Blog de Mathilde

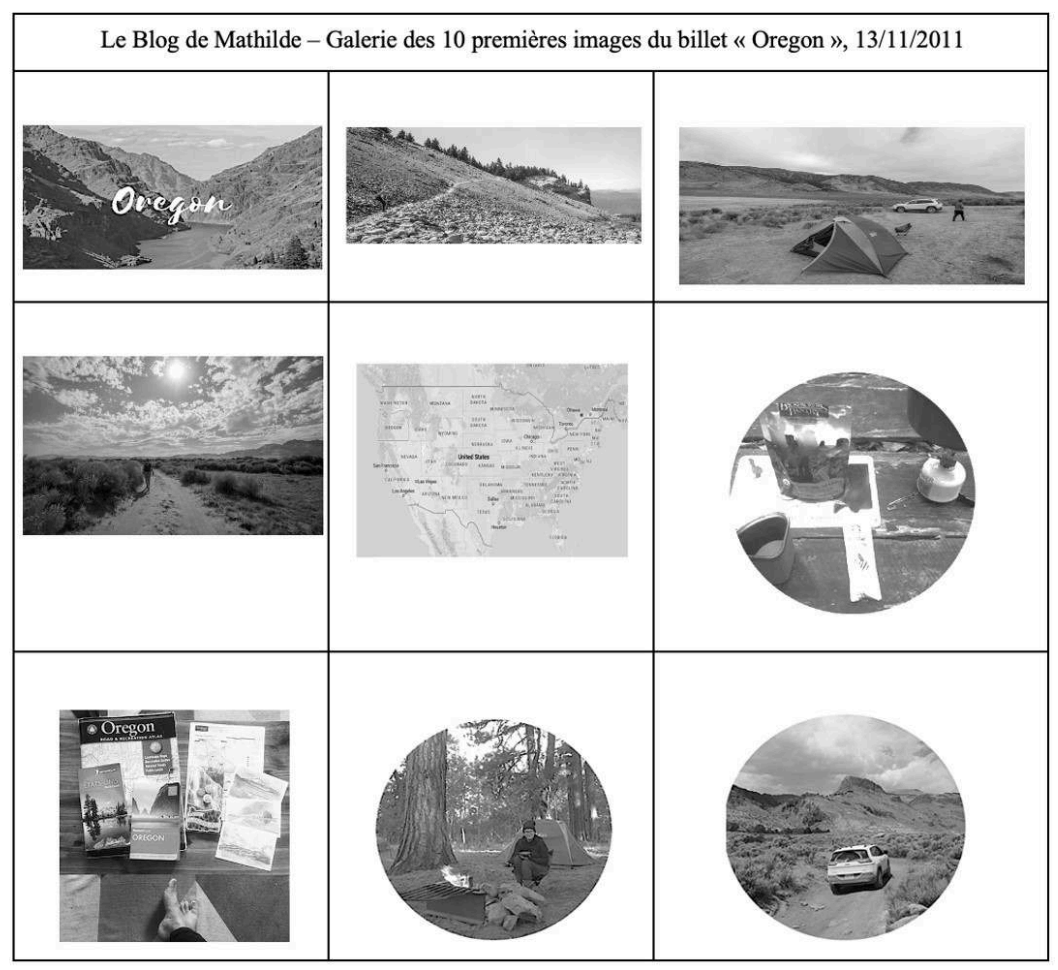

Source : https://www.maathiildee.com/road-trip-oregon-etats-unis/ 
Illustration 8b. Exemple d'analyse de l'image d'accroche d'un billet de blog extrait du premier corpus selon les critères de la grammaire visuelle de Kress et van Leeuwen (2006)

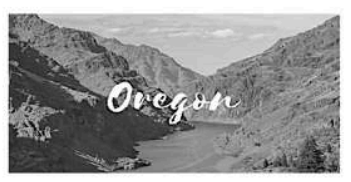

« Les participants représentés » (Kress et van Leeuwen, 2006 : p. 47) ne sont pas humains. Ils sont au nombre de deux : le titre de la photographie en premier plan et le paysage en arrière-plan. Les deux plans se superposent l'un sur l'autre.

Les relations entre les participants représentés se définissent, $d$ 'une part, par les lignes vectorielles qu organisent la structure visuelle de l'image et, d'autre part, par la superposition des plans (op. cit. : p. 59). Ainsi, selon cette organisation, le titre est mis en scène comme étant l'acteur d'une action qui relierail le premier plan à l'arrière-plan suivant une ligne tracée par la rivière. La structure de la photo semble indiquer que le titre est l'acteur d'un processus narratif dont l'objectif est d'atteindre le paysage qui, lui, est présenté en arrière-plan. La superposition des deux plans horizontaux indique une relation quasi définitoire entre l'acteur et son objectir.

On note que l'analyse des relations entre les participants représentés (présents sur la photo) et l'observateur (le blogueur, le photographe ou encore le lecteur du blog) est distincte selon que le regard se concentre sur le message ou sur le paysage, sur le premier plan ou sur l'arrière-plan. Ainsi, si l'on privilégie le premier plan, les analyses du frontal angle (op. cit. : p. 145) et du cadrage (op. cit. : p. 124) suggèrent l'existence d'une relation d'égal à égal (l'angle frontal se situe à hauteur des yeux du spectateur), voire de proximité entre l'acteur (le titre) et l'observateur (le blogueur ou le spectateur). Or, si l'analyste privilégie l'arrière-plan, la relation qui s'établit alors entre l'observateur et le paysage photographié est différente de celle identifíée lors de la description du premier plan : le cadrage de la photo est lointain, ce qui laisse supposer que le paysage n'est présent que dans une visée contemplative puisqu'il se situe hors de portée de l'observateur.

Les relations de pouvoir sont représentées par les axes verticaux (op. cit. : p. 140). Ici, l'observateur se voit offrir une possibilité d'action sur l'objectif à atteindre (le paysage) puisque l'angle de vue du haut vers le bas lui attribue un certain pouvoir (lbid.).

Schẻma vectoriel (flèches) et ligne de regard de l'observateur (trait horizontal):

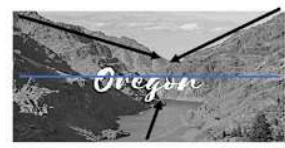

Source de l'image : https///www.maathiildee.com/road-trip-oregon-etats-unis/

Illustration 9. Des sites touristiques photographiés avant et pendant la période d'observation

Avant mars 2020 :

\begin{tabular}{|c|c|c|}
\hline $\begin{array}{c}\text { Le Sac à Dos - « Mon trek en } \\
\text { Himalaya avec Dawa Sherpa ", } \\
2015\end{array}$ & $\begin{array}{c}\text { Maman Voyage }- \text { « Visiter Portsmouth en } \\
\text { famille », } 9 \text { février 2020 }\end{array}$ & $\begin{array}{c}\text { The Daydreameuse - « Dans les rues } \\
\text { de Bruxelles », } 18 \text { février } 2016\end{array}$ \\
\hline & & \\
\hline
\end{tabular}

De mars 2020 à janvier 2021 :

\begin{tabular}{|l|c|l|}
\hline $\begin{array}{l}\text { Bestjobers - « } 6 \text { idées week-end } \\
\text { pour visiter les châteaux de la } \\
\text { Loire \#, 13 août 2020 }\end{array}$ & $\begin{array}{c}\text { Un Couple en Vadrouille - « Visiter } \\
\text { Gran Canaria, une des îles Canaries », } \\
18 \text { juin 2020 }\end{array}$ & $\begin{array}{l}\text { Japon Secret « Aogashima: l'île au } \\
\text { double volcan », 13 juin 2020 }\end{array}$ \\
\hline & &
\end{tabular}


Illustration 10. Exemples d'images de guides et de publicités touristiques

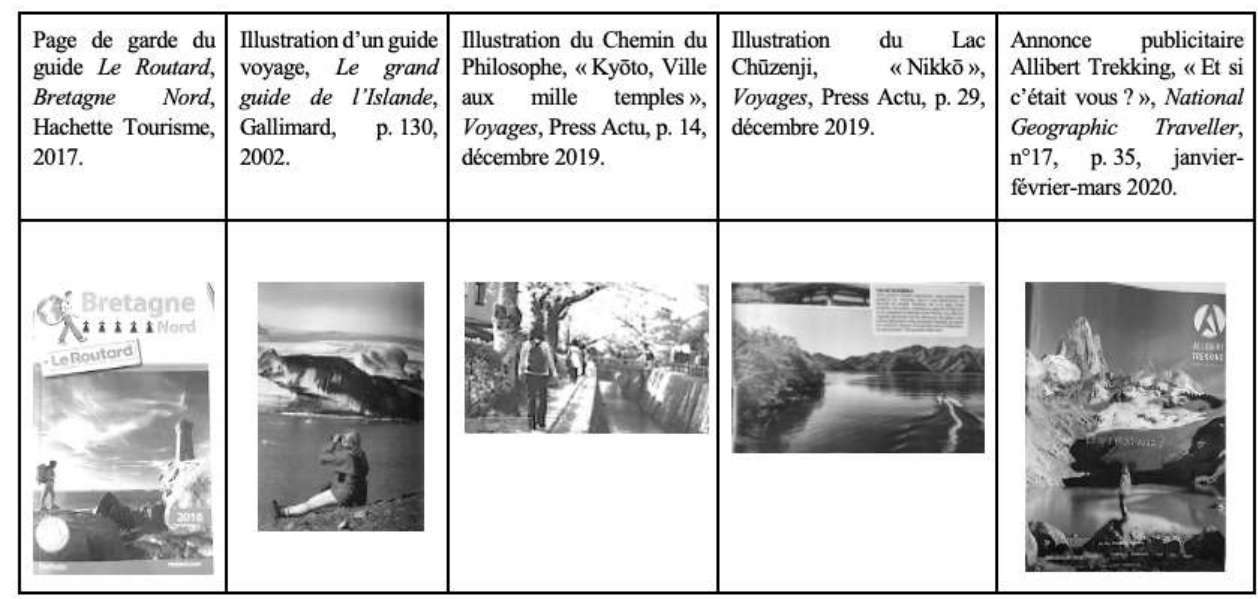

Illustration 11. Exemples de photos « de dos », extraits du corpus 2

\begin{tabular}{|c|c|c|}
\hline Bestjobers & GaijinJapan & Un Couple en Vadrouille \\
\hline & & \\
\hline $\begin{array}{l}\text { Date : } 13 / 08 / 2020 \\
\text { https://www.bestjobersblog.com/idees- } \\
\text { week-end-visiter-chateaux-de-la-loire/ }\end{array}$ & $\begin{array}{l}\text { hattps:/www.gaijinjapan.org/voyage-au- } \\
\text { japon-2020-possible-ou-pas/ }\end{array}$ & $\begin{array}{l}\text { Date : } 18 / 06 / 2020 \\
\text { https://www.uncoupleenvadrouille.fr/visiter- } \\
\text { gran-canaria-une-des-iles-canaries/ }\end{array}$ \\
\hline
\end{tabular}


Illustration 12. Extraits de photos avant 2019 et après 2020, Un Couple en Vadrouille

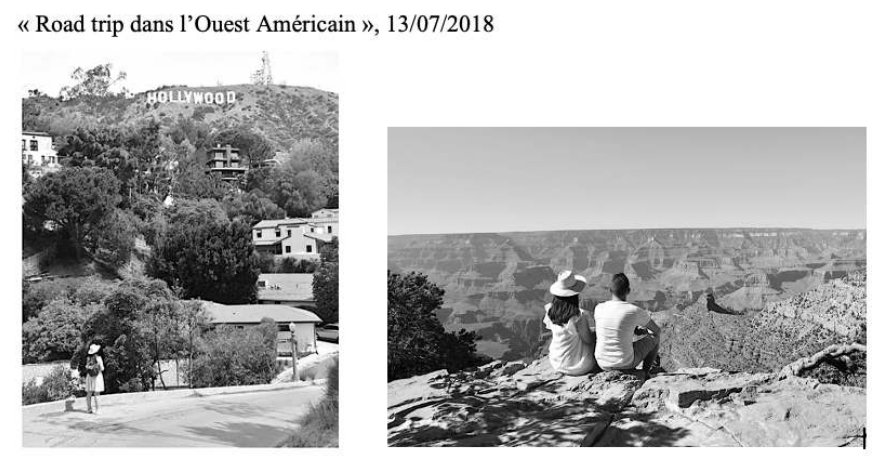

https://www.uncoupleenvadrouille.fr/road-trip-ouest-americain/

«Visiter Verdun », 09/12/2020 et « Une semaine à Dubaï \& Abu Dhabi », 18/09/2020
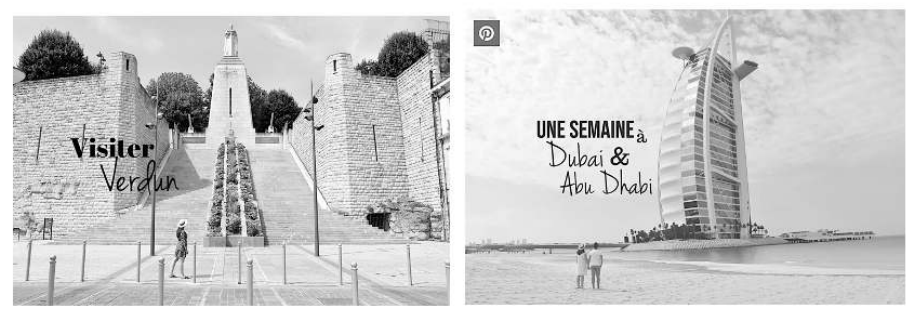

https://www.uncoupleenvadrouille.fr/visiter-verdun/

https://www.uncoupleenvadrouille.fr/une-semaine-a-dubai-et-abu-dhabi/

Illustration 13. « Point Covid-19 en Martinique (situation mi-octobre 2020) », Bestjobers, 13 septembre 2020

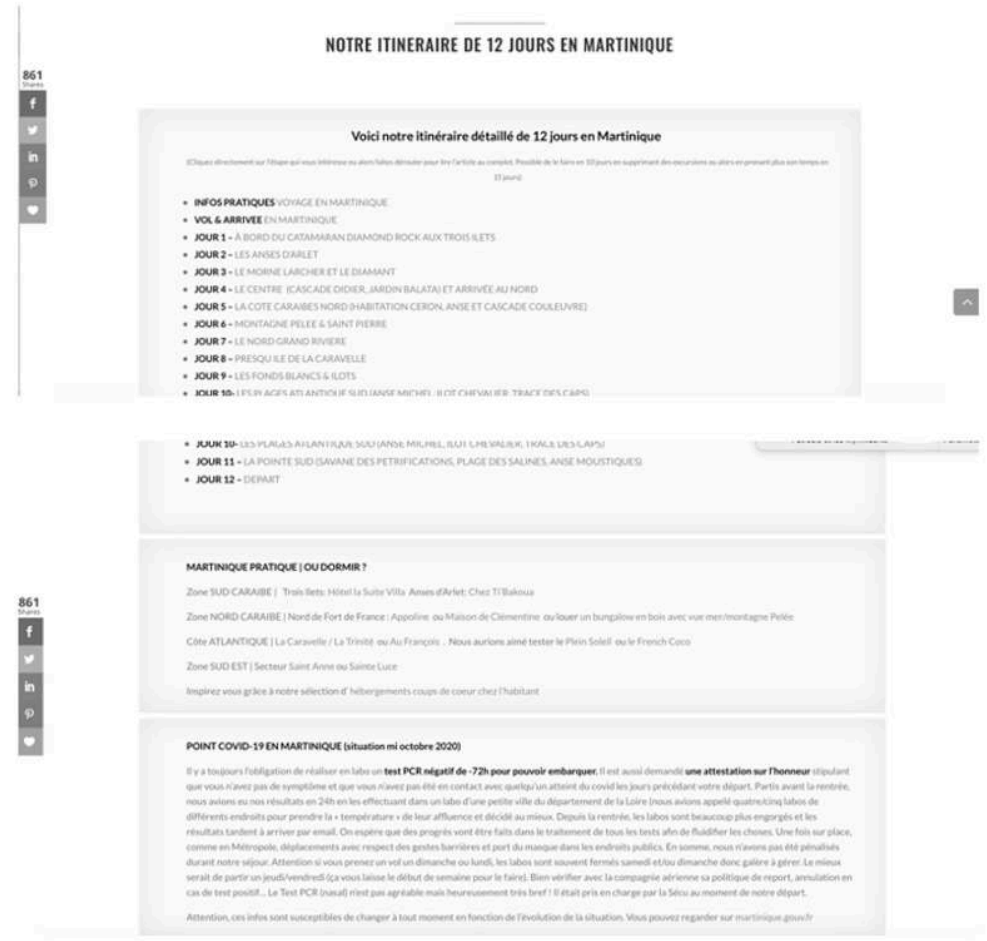

Source : https://www.bestjobersblog.com/que-faire-en-martinique-itineraire-conseils/, 13 septembre 2020 


\section{NOTES}

1. Les représentations individuelles témoignent de l'incorporation des représentations collectives partagées et reproduites dans une société donnée (Moscovici, 2003). Les représentations collectives construisent une organisation du réel renfermant des connaissances sur le monde qui permettent aux individus de juger et de valoriser les objets sociaux de cette réalité (Charaudeau, 1997).

2. C'est avec un infini respect pour l'ensemble des personnes et des travailleurs affectés par la crise sanitaire que nous présentons ce travail qui répond modestement à une démarche de documentation des premiers effets sociétaux de la pandémie.

3. Le terme de paradigme chez Kuhn désigne «l'ensemble de croyances, de valeurs reconnues et de techniques qui sont communes aux membres d'un groupe donné » (Kuhn, 1972, p. 207).

4. Les stratégies discursives de persuasion (légitimation, crédibilité et captation) répondent à des visées d'incitation à faire qu'on emploie «dans une situation où l'on a besoin de quelqu'un d'autre [individu ou public] pour réaliser son projet, et qu'on n'a pas autorité absolue sur cet autre pour l'obliger à agir dans un certain sens » (Charaudeau, 2009, p. 8).

5. Le champ discursif est « un espace où l'on travaille sans cesse à construire et à préserver des identités énonciatives dont il faut montrer la légitimité » et où s'affronte une multitude d'acteurs pour «obtenir ou préserver une autorité énonciative» en inscrivant «leurs paroles à une certaine place » (Maingueneau, 2019, p. 189).

6. «L'objet de l'analyse du discours, ce n'est ni les fonctionnements textuels, ni la situation de communication, mais ce qui les noue à travers un dispositif d'énonciation qui relève à la fois du verbal et de l'institutionnel » (Maingueneau, 2014, p. 44).

7. On emprunte à Bourdieu la notion de champ (Bourdieu, 1976) que l'on applique au domaine des voyages réalisés dans une optique de quête de plaisir (Urbain, 2018).

8. Les genres discursifs sont des « dispositifs de communication " (Maingueneau, 2013, p. 50) qui se caractérisent par des « régularités et des variabilités discursives » (Münchow, 2007, p. 126).

9. https://www.youtube.com/channel/UCyOphwnua5w-ugqL9Rdownw

10. https://www.instagram.com/lilyrose/

11. https://www.votretourdumonde.com

12. Le terme " communautés» désigne des groupes réunissant des individus «qui interagissent entre eux, partagent des liens d'attachement fort autour de passions et expériences communes » (Vaublanc, 2019, p. 237-239).

13. Les schèmes constituent «une catégorie de connaissances stockées en mémoire » et «ils permettent entre autres d'inférer des informations ou des éléments de situation qui n'ont pas été explicités dans le message, alors qu'ils sont nécessaires à sa compréhension » (Rossi, 2005, p. 202). 14. On citera la revendication d'un des blogueurs du corpus pour illustrer ce propos: «Japon Secret est un blog sur le Japon alternatif, l'exploration de lieux en dehors des sentiers battus. Le contenu est réalisé avec passion par Jordy Meow et n'est pas sponsorisé. Bonne visite :) » (Japon Secret, sans date).

15. Japon Secret.

16. Le Sac à Dos.

17. Villages du Japon, livre réalisé par Japon Secret.

18. C'est le cas du blog Le Sac à Dos qui précise ses conditions pour réaliser des partenariats : «Je ne fais pas de posts sponsorisés, échanges de liens, insertion de liens ou autres publicités du même style. Toutes les demandes en ce sens seront supprimées. (...) Si vous souhaitez mettre en avant une expérience ou une destination à travers un blog trip, je suis prêt à en discuter » (Le Sac à Dos, non daté). 
19. Selon l'enquête annuelle de l'agence de marketing Reech, seuls « $15 \%$ [des influenceurs] sont des créateurs de contenus à temps plein » et $6 \%$ "gagnent plus de 20000 euros par an » : https:// www.reech.com/fr/influence-etude-reech-2021, consulté le 14 mars 2021.

20. Les huit blogs du corpus auquel nous avons ajouté un site populaire de la blogosphère francophone (un des sites ayant disparu durant la période observée), soit un ajout de 68 billets et 6 vidéos aux éléments du premier corpus.

21. À la date de publication de cet article, l'organisation mondiale du commerce indique que « les arrivées sont toujours inférieures de $76 \%$ aux niveaux prépandémiques » et que les recettes du tourisme international pourraient atteindre en 2021 « moins de la moitié du niveau enregistré en 2019 qui était de 1700 milliards d'USD » (https://www.unwto.org/fr/news/le-tourisme-mondialconnait-une-embellie-au-troisieme-trimestre-mais-la-reprise-reste-fragile, consulté le 2 décembre 2021).

22. Organisation mondiale du tourisme, https://www.unwto.org/news/2020-worst-year-intourism-history-with-1-billion-fewer-international-arrivals, consulté le 9 mars 2021.

23. On note, sur ce point, l'intérêt d'un dossier consacré aux effets de la crise sanitaire sur les économies caribéennes, paru en août 2021 (https://journals.openedition.org/etudescaribeennes/ 21099) ; la publication en 2020 de Demeyer sur les conditions de travail et la précarité dans le secteur touristique (https://journals.openedition.org/teoros/7457). Pour une approche gestionnaire, on consultera les travaux interrogeant les répercussions de la Covid-19 sur les productions des travel influencers exerçant sur Instagram (https://www.sciencedirect.com/ science/article/pii/S0261517721001734).

24. On désignera par la suite les auteurs de blogs voyage par le nom de leur blog.

25. Dans la transcription, on note «: " pour un allongement vocalique et «/ " pour une courte pause.

26. La transcription du texte est fidèle à celle du blog. Les éventuelles erreurs d'orthographe, de syntaxe ou de grammaire ne sont pas corrigées.

\section{RÉSUMÉS}

Dans ce travail, on documente l'évolution des pratiques sociolangagières de huit blogueurs populaires traitant du thème du voyage durant les premiers mois de la pandémie dans le cadre théorique de l'analyse du discours. La confrontation de données plurisémiotiques réunies entre mars 2020 et janvier 2021 aux résultats d'une étude antérieure portant sur les modalités de l'invitation au voyage dans ces mêmes travel blogs pointe comment, dans le contexte de l'effondrement de la mobilité d'agrément, les auteurs de notre corpus développent de nouvelles stratégies de captation du public tout en maintenant dans leurs productions les signes de l'envie du monde.

Within the theoretical framework of discourse analysis, we show how the Covid-19 pandemic has impacted the social and discursive practices of eight popular travel bloggers. In a context of complete leisure mobility shut down, how have touristic representations been changed? How have authors found a way to maintain in their productions the desire to travel? In this work, we compare textual and semiotic data gathered between March 2020 and January 2021 to the results of a previous study based on these same travel blogs between 2016 and 2019. After having addressed the discursive image of the authors who stand out as travel influencers on social 
networks, we offer a reminder of the main "imaginaries" that shape positions in the discursive field of tourism. We then describe how bloggers, in this specific study, are reinventing, or not, their publication and fundraising habits to tackle the consequences of the health situation. We end by reporting on the linguistic and visual practices they used to maintain travel rhetoric wonder effects.

\section{INDEX}

Keywords : travel influencers, travel blogs, discourse analysis, tourism discourses, pandemic, Covid-19

Mots-clés : influenceurs voyage, blogs voyage, analyse du discours, discours du tourisme, pandémie, Covid-19

\section{AUTEUR}

\section{EUGÉNIE PEREIRA COUTTOLENC}

Sciences du langage - Analyse du discours

Doctorante et chargée d'enseignements - Université de Paris

Eugenie.pereira[at]etu.u-paris.fr 